Supporting Information for

\title{
Allylpalladium "Umpolung” in the Three-Component Coupling Synthesis of Homoallylic Amines
}

\author{
Chad D. Hopkins and Helena C. Malinakova* \\ Department of Chemistry, University of Kansas, 1251 Wescoe Hall Drive, \\ Lawrence, KS 66045-7582 \\ hmalina@ku.edu
}

\section{Table of Contents}

\author{
Page \\ General Experimental $\quad$ S-2 \\ Synthesis and Complete Characterization Data for Compounds 4a-d and 5-12, 13a, 13b, 14a, 14b $\quad$ S-3 \\ Coupling to iminoacetates bearing other $N$-protecting groups (Table S-1) (Reference 11) S-20 \\ Overview of optimization studies in the synthesis of amine 4a (Tables S-2) (Reference 14) S-20 \\ Formation of amine 4a under Pd(0) catalysis (Reference 15) S-22 \\ NOE ${ }^{1} \mathrm{H}$ NMR studies on tetrahydropyridines 14a and $\mathbf{1 4 b} \quad S-22$ \\ $\begin{array}{ll}\text { References } & \text { S-24 }\end{array}$ \\ ${ }^{1} \mathrm{H}$ NMR spectra for the byproduct fraction from Entry 5 (Table 2) $\quad$ S-25 \\ Copies of ${ }^{1} \mathrm{H}$ NMR and ${ }^{13} \mathrm{C}$ NMR spectra for Compounds 4a-d and 5-12, 13a, 13b, 14a, 14b $\quad$ S-26 \\ Results of X-ray crystallographic analysis on amine $10 \quad$ S-63
}




\section{General Experimental}

Unless otherwise indicated, all NMR data were collected at room temperature in $\mathrm{CDCl}_{3}$ with internal $\mathrm{CHCl}_{3}$ as the reference $\left(\square 7.26 \mathrm{ppm}\right.$ for ${ }^{1} \mathrm{H}$ and $77.00 \mathrm{ppm}$ for $\left.{ }^{13} \mathrm{C}\right)$. IR spectra were measured as thin films on salt $(\mathrm{NaCl})$ plates. Melting points are uncorrected and were taken in open capillary tubes. MS were measured under electrospray ionization $\left(\mathrm{ES}^{+}\right)$conditions. Analytical thin-layer chromatography (TLC) was carried out on commercial Merck silica gel 60 plates, $250 \square \mathrm{m}$ thickness, with fluorescent indicator (F254) or stained with aqueous $\mathrm{KMnO}_{4}$ solution. Column chromatography was performed with 32-63 $\square \mathrm{m}$ silica gel (Sorbent). Tetrahydrofuran (THF) was freshly distilled from sodium/benzophenone. 1,2-Dichloroethane, methylene chloride, DMF, AcCN, MeOH, and toluene were kept over $3 \AA$ (8-12 mesh) molecular sieves under an atmosphere of dry argon; other solvents were used as received. Unless otherwise specified, all reactions were carried out under an atmosphere of dry argon in oven-dried (at least $6 \mathrm{~h}$ at $140{ }^{\circ} \mathrm{C}$ ) glassware. $p$-Methoxyphenylboronic acid was purchased from Aldrich, purified by recrystallization from water, and dried under vacuum for at least $16 \mathrm{~h}$. 1,2-nonadiene ${ }^{1}$ and $(+)-\left(3,2,10-\square\right.$-pinene)palladium(II) chloride $\mathbf{1}^{2}$ were prepared according to the indicated literature protocols. All imines were prepared according to a modified literature procedure ${ }^{3}$ by condensation of a $1: 1$ mixture of aldehyde and amine in benzene $\left(65^{\circ} \mathrm{C}\right)$ or refluxing toluene in the presence of activated $3 \AA$ ( $8-12$ mesh) molecular sieves for $24 \mathrm{~h}$ followed by filtration through celite and removal of solvent under vacuum to afford pure imines that were used immediately. Other materials were used as received from commercial suppliers. 
General protocol for the preparation of homoallylic amines 4 a-d and 5-12. A solution of 1,2-nonadiene (5.0 equiv) and imine (1.0 equiv) in dry THF (5 mL) was injected into a reaction vessel containing the solid reagents including the boronic acid (2.0 equiv), palladium catalyst ( 0.1 equiv of $\mathrm{Pd})$, phosphine ligand ( 0.1 equiv) and $\mathrm{CsF}$ (4.0 equiv). In situations when the imine was a solid, it was added along with the other solid reagents. The reaction mixture was then stirred at the indicated temperature under argon for $24 \mathrm{~h}$. Water $(20 \mathrm{~mL})$ was added, and the mixture was extracted with ether $(4 \mathrm{x}$ $20 \mathrm{~mL})$. Organic extracts were dried $\left(\mathrm{MgSO}_{4}\right)$, and the solvents were removed under reduced pressure to afford crude products that were separated by flash chromatography over silica eluting with EtOAc/Hexanes mixtures to yield pure amines 4a-d and 5-12 as colorless to yellow oils.

Ethyl-3-(1-hexyl)-4-(p-methoxyphenyl)-2-( $N$-phenylamino)-4-pentenoate (major diastereomer) (4a). Treatment of $p$-methoxyphenylboronic acid $(0.070 \mathrm{~g}, 0.461 \mathrm{mmol}$, 2.0 equiv), $\mathrm{CsF}$ (0.140 g, $0.921 \mathrm{mmol}, 4.0$ equiv), ethyl $N$-phenyliminoacetate $(0.041 \mathrm{~g}$, $0.230 \mathrm{mmol}, 1.0$ equiv) and 1,2-nonadiene ( $0.143 \mathrm{~g}, 1.15 \mathrm{mmol}, 5.0$ equiv) with (+)(3,2,10- $\square$-pinene)palladium(II) chloride 1 ( $0.006 \mathrm{~g}, 0.011 \mathrm{mmol}, 0.05$ equiv) and $\mathrm{P}(o$ Tol $)_{3}(0.007 \mathrm{~g}, 0.023 \mathrm{mmol}, 0.1$ equiv $)$ at $\mathrm{rt}$ according to the general procedure described above followed by flash chromatography over silica eluting with EtOAc/hexane $(1: 20)$ afforded $4 \mathbf{a}(0.058 \mathrm{~g}, 61 \%)$ as a mixture of inseparable diastereomers (dr $7: 1)$ as a colorless oil.

Analytical data for 4a: $\mathrm{R}_{f}=0.56($ EtOAc/hexane $1: 5) ;{ }^{1} \mathrm{H} \mathrm{NMR}\left(500 \mathrm{MHz}, \mathrm{CDCl}_{3}\right) \square$ $7.33(\mathrm{~d}, J=9.0 \mathrm{~Hz}, 0.25 \mathrm{H}), 7.23(\mathrm{~d}, J=8.5 \mathrm{~Hz}, 1.75 \mathrm{H}), 7.13(\mathrm{t}, J=10 \mathrm{~Hz}, 2 \mathrm{H}), 6.88$ $(\mathrm{d}, J=11 \mathrm{~Hz}, 0.25 \mathrm{H}), 6.84(\mathrm{~d}, J=7.0 \mathrm{~Hz}, 1.75 \mathrm{H}), 6.72(\mathrm{t}, J=7.5 \mathrm{~Hz}, 1 \mathrm{H}), 6.55(\mathrm{~d}, J=$ 8.0 Hz, $2 \mathrm{H})$, 5.34, (s, 0.12 H), 5.32 (s, $0.88 \mathrm{H}), 5.13$ (s, $0.88 \mathrm{H}), 5.11(\mathrm{~s}, 0.12 \mathrm{H}), 4.15$ (s 
br, $1 \mathrm{H}), 4.11(\mathrm{~d}, J=6.0 \mathrm{~Hz}, 0.12 \mathrm{H})), 4.0(\mathrm{~d}, J=6.0 \mathrm{~Hz}, 0.88 \mathrm{H}), 4.10-4.03(\mathrm{~m}, 0.25 \mathrm{H})$, 3.91-3.73 (m, $1.75 \mathrm{H}), 3.80(\mathrm{~s}, 3 \mathrm{H}), 3.12(\mathrm{dt}, J=9.5 \mathrm{~Hz}, 5.5 \mathrm{~Hz}, 0.88 \mathrm{~Hz}),(3.0$ (ddd, $J=$ $10.0 \mathrm{~Hz}, 6.0 \mathrm{~Hz}, 4.0 \mathrm{~Hz}, 0.12 \mathrm{H}), 1.68-1.58$ (m, $2 \mathrm{H}), 1.50-1.39$ (m, $1 \mathrm{H}), 1.37-1.23$ (m, 7 $\mathrm{H}), 1.17(\mathrm{t}, J=5.0 \mathrm{~Hz}, 0.37 \mathrm{H}), 1.10(\mathrm{t}, J=7.5 \mathrm{~Hz}, 2.63 \mathrm{H}), 0.87(\mathrm{t}, J=7.0 \mathrm{~Hz}, 3 \mathrm{H}) ;{ }^{13} \mathrm{C}$ NMR (125 MHz, $\left.\mathrm{CDCl}_{3}\right) \square 173.1$ (173.0), 159.1, 147.5 (147.6), 147.1 (147.2), 134.7 (134.9), 129.2 (129.1) (two carbons), 128.3 (128.0) (two carbons), 118.1 (118.2), 114.9, 113.6 (113.9) (two carbons), 113.5 (113.8) (two carbons), 60.7 (60.9), 59.3 (60.8), 55.3 (55.7), 47.3 (47.0), 31.7 (31.6), 30.9 (30.4), 29.3 (29.4), 27.3 (27.1), 22.6, 14.0 (14.2), 13.9, signals for the minor diastereomer are given in parentheses; IR (neat, $\left.\mathrm{cm}^{-1}\right) 3384(\mathrm{w}$ br), 1731 (s), 1604 (s), 1510 (s); HRMS (ES ${ }^{+}$calcd for $\mathrm{C}_{26} \mathrm{H}_{36} \mathrm{NO}_{3}\left(\mathrm{M}+\mathrm{H}^{+}\right)$, 410.2695, found 410.2684 .

\section{Ethyl-3-(1-hexyl)-4-(p-methoxycarbonylphenyl)-2-( $N$-phenylamino)-4-pentenoate}

(major diastereomer) (4b). Treatment of p-methoxycarbonylphenylboronic acid (0.083 g, $0.461 \mathrm{mmol}, 2.0$ equiv), $\mathrm{CsF}(0.140 \mathrm{~g}, 0.921 \mathrm{mmol}, 4.0$ equiv), ethyl $\mathrm{N}$ phenyliminoacetate $(0.041 \mathrm{~g}, 0.230 \mathrm{mmol}, 1.0$ equiv) and 1,2-nonadiene ( $0.143 \mathrm{~g}, 1.15$ mmol, 5.0 equiv) with $(+)-(3,2,10$ - - -pinene)palladium(II) chloride 1 (0.006 g, 0.011 mmol, 0.05 equiv $)$ and $\mathrm{P}(o-\mathrm{Tol})_{3}(0.007 \mathrm{~g}, 0.023 \mathrm{mmol}, 0.1$ equiv $)$ at $\mathrm{rt}$ according to the general procedure described above followed by flash chromatography over silica eluting with EtOAc/hexane $(1: 20)$ afforded $\mathbf{4 b}(0.077 \mathrm{~g}, 76 \%)$ as a mixture of inseparable diastereomers $(\mathrm{dr} 13: 1)$ as a yellow oil. 
Analytical data for $4 \mathbf{b}: \mathrm{R}_{f}=0.47(\mathrm{EtOAc} / \mathrm{hexane} 1: 5) ;{ }^{1} \mathrm{H} \mathrm{NMR}\left(400 \mathrm{MHz}, \mathrm{CDCl}_{3}\right) \square$ $8.13(\mathrm{~d}, J=8.4 \mathrm{~Hz}, 0.14 \mathrm{H}), 7.98(\mathrm{~d}, J=8.4 \mathrm{~Hz}, 1.86 \mathrm{H}), 7.50(\mathrm{~d}, J=8.4 \mathrm{~Hz}, 0.14 \mathrm{H})$, $7.36(\mathrm{~d}, J=8.4 \mathrm{~Hz}, 1.86 \mathrm{H}), 7.14(\mathrm{t}, J=12.0 \mathrm{~Hz}, 2 \mathrm{H}), 6.72(\mathrm{t}, J=7.2 \mathrm{~Hz}, 1 \mathrm{H}), 6.55(\mathrm{~d}$, $J=8.0 \mathrm{~Hz}, 2 \mathrm{H}), 5.49(\mathrm{~s}, 0.07 \mathrm{H}), 5.44(\mathrm{~s}, 0.93 \mathrm{H}), 5.29(\mathrm{~s}, 1 \mathrm{H}), 4.20-4.05(\mathrm{~s}$ br. $1 \mathrm{H})$, 4.12-4.07 (m, 0.14 H), $4.02(\mathrm{~d}, J=6.0 \mathrm{~Hz}, 1 \mathrm{H}), 3.93(\mathrm{~s}, 0.21 \mathrm{H}), 3.92(\mathrm{~s}, 2.79 \mathrm{H}), 3.88-$ $3.78(\mathrm{~m}, 0.93 \mathrm{H}), 3.77-3.62(\mathrm{~m}, 0.93 \mathrm{H}), 3.16(\mathrm{dd}, J=14.0 \mathrm{~Hz}, 6.0 \mathrm{~Hz}, 0.93 \mathrm{H}), 3.11-3.05$ (m, 0.07 H), 1.71-1.59 (m, 2 H), 1.52-1.45 (m, 1 H), 1.39-1.27 (m, 7 H), $1.16(\mathrm{t}, J=7.2$ $\mathrm{Hz}, 0.21 \mathrm{H}), 1.06(\mathrm{t}, J=7.2 \mathrm{~Hz}, 2.79 \mathrm{H}), 0.88(\mathrm{t}, J=6.8 \mathrm{~Hz}, 3 \mathrm{H}) ;{ }^{13} \mathrm{C}$ NMR $(125 \mathrm{MHz}$, $\left.\mathrm{CDCl}_{3}\right) \square$ 172.9, 166.8 (166.9), 147.4, (147.8), 147.1 (147.2), 146.8 (146.9), 129.5 (129.8) two carbons, 129.2 (129.1) two carbons, 129.0, 127.2 two carbons, 118.4, 116.9, 113.4 (113.7) two carbons, 60.8 (60.9), 59.3, 52.1 (52.2), 47.2 (46.9), 31.7 (31.60), 30.9 (30.5), 29.3, 27.3 (27.1), 22.6, 14.0, 13.9, signals for the minor diastereomer are given in parentheses; IR (neat, $\mathrm{cm}^{-1}$ ) 3380 (w br), 1724 (s), $1602(\mathrm{~m})$; HRMS (ES $)$ calcd for $\mathrm{C}_{27} \mathrm{H}_{36} \mathrm{NO}_{4}\left(\mathrm{M}+\mathrm{H}^{+}\right), 438.2645$, found 438.2653 .

\section{Ethyl-3-(1-hexyl)-4-(p-methoxyphenyl)-2-( $N$-p-methoxyphenylamino)-4-pentenoate}

(major diastereomer) (4c). Treatment of $p$-methoxyphenylboronic acid $(0.069 \mathrm{~g}, 0.452$ mmol, 2.0 equiv), CsF $(0.137 \mathrm{~g}, 0.905 \mathrm{mmol}, 4.0$ equiv), ethyl $N$-( $p$ methoxyphenyl)iminoacetate $(0.043 \mathrm{~g}, 0.225 \mathrm{mmol}, 1.0$ equiv) and 1,2-nonadiene ( 0.140 $\mathrm{g}, 1.12 \mathrm{mmol}, 5.0$ equiv) with $\mathrm{Pd}(\mathrm{OAc})_{2}(0.005 \mathrm{~g}, 0.023 \mathrm{mmol}, 0.1$ equiv $)$ and $\mathrm{H}[\mathrm{P}(t-$ $\left.\mathrm{Bu})_{3}\right] \mathrm{BF}_{4}(0.007 \mathrm{~g}, 0.023 \mathrm{mmol}, 0.1$ equiv $)$ at $\mathrm{rt}$ according to the general procedure described above followed by flash chromatography over silica eluting with EtOAc/hexane $(1: 12)$ afforded $4 \mathbf{c}(0.053 \mathrm{~g}, 54 \%)$ as a mixture of inseparable diastereomers (dr $10: 1)$ as a colorless oil. 
Analytical data for 4c: $\mathrm{R}_{f}=0.59($ EtOAc/hexane $1: 3) ;{ }^{1} \mathrm{H}$ NMR $\left(400 \mathrm{MHz}, \mathrm{CDCl}_{3}\right)$ $7.37(\mathrm{~d}, J=6.8 \mathrm{~Hz}, 0.18 \mathrm{H}), 7.33(\mathrm{~d}, J=6.8 \mathrm{~Hz}, 0.18 \mathrm{H}), 7.23(\mathrm{~d}, J=8.8 \mathrm{~Hz}, 1.82 \mathrm{H})$, $6.89(\mathrm{~d}, J=8.4 \mathrm{~Hz}, 0.18 \mathrm{H}), 6.84(\mathrm{~d}, J=6.4 \mathrm{~Hz}, 1.82 \mathrm{H}), 6.73(\mathrm{~d}, J=8.8 \mathrm{~Hz}, 1.82 \mathrm{H})$, $6.54(\mathrm{~d}, J=9.2 \mathrm{~Hz}, 2 \mathrm{H}), 5.34(\mathrm{~s}, 0.09 \mathrm{H}), 5.31(\mathrm{~s}, 0.91 \mathrm{H}), 5.13(\mathrm{~s}, 0.91 \mathrm{H}), 5.11(\mathrm{~s}, 0.09$ H), 4.08-3.96 (m, 0.18 H), 3.90 (s br $1 \mathrm{H}), 3.86-3.73(\mathrm{~m}, 2.82 \mathrm{H}), 3.82(\mathrm{~s}, 0.27 \mathrm{H}), 3.80$ $(\mathrm{s}, 2.73 \mathrm{H}), 3.79(\mathrm{~s}, 0.27 \mathrm{H}), 3.72(\mathrm{~s}, 2.73 \mathrm{H}), 3.10$ (dt, $J=9.2 \mathrm{~Hz}, 4.8 \mathrm{~Hz}, 0.91 \mathrm{H}), 3.03$ (ddd, $J=10.4 \mathrm{~Hz}, 6.0 \mathrm{~Hz}, 4.0 \mathrm{~Hz}, 0.09 \mathrm{H}), 1.68-1.56$ (m, $2 \mathrm{H}), 1.52-1.41$ (m, $1 \mathrm{H}), 1.37-$ $1.27(\mathrm{~m}, 7 \mathrm{H}), 1.14(\mathrm{t}, J=6.8 \mathrm{~Hz}, 0.27 \mathrm{H}), 1.07(\mathrm{t}, J=7.2 \mathrm{~Hz}, 2.73 \mathrm{H}), 0.87(\mathrm{t}, J=6.8$ $\mathrm{Hz}, 3 \mathrm{H}) ;{ }^{13} \mathrm{C}$ NMR (125 MHz, $\left.\mathrm{CDCl}_{3}\right) \square 173.4$ (173.1), 159.0 (158.9), 152.6 (152.7), 147.5 (147.8), 141.2 (141.3), 134.8 (135.0), 128.3 (127.9, 126.3) two carbons, 115.3 (115.5) two carbons, 114.9 (113.9), 114.7 (114.6) two carbons, $113.4(113.7,113.6)$ two carbons, 60.7 (62.3), 60.6 (60.8), 55.7 (55.5), 55.2 (55.3), 47.3 (47.0), 31.7 (31.6), 31.0 (30.7), 29.3 (29.4), 27.3 (27.2), 22.6 (25.5), 14.0 (14.2), 13.9 (14.0), signals for the minor diastereomer are given in parentheses; IR (neat, $\mathrm{cm}^{-1}$ ) 3384 (w br), 1731 (s), 1606 (s); HRMS (ES $)$ calcd for $\mathrm{C}_{27} \mathrm{H}_{38} \mathrm{NO}_{4}\left(\mathrm{M}+\mathrm{H}^{+}\right)$, 440.2801, found 440.2792.

\section{Ethyl-3-(1-hexyl)-4-(p-methoxycarbonylphenyl)-2-( $N$-p-methoxyphenylamino)-4- pentenoate (major diastereomer) (4d).}

Treatment of $p$-methoxycarbonylphenylboronic acid ( $0.081 \mathrm{~g}, 0.450 \mathrm{mmol}, 2.0$ equiv), CsF (0.137 g, $0.900 \mathrm{mmol}, 4.0$ equiv), ethyl $N$-( $p$-methoxyphenyl)iminoacetate $(0.043 \mathrm{~g}$, $0.225 \mathrm{mmol}, 1.0$ equiv) and 1,2-nonadiene (0.140 g, $1.13 \mathrm{mmol}, 5.0$ equiv) with $\mathrm{Pd}(\mathrm{OAc})_{2}\left(0.005 \mathrm{~g}, 0.023 \mathrm{mmol}, 0.1\right.$ equiv) and $\mathrm{H}\left[\mathrm{P}(t-\mathrm{Bu})_{3}\right] \mathrm{BF}_{4}(0.007 \mathrm{~g}, 0.023 \mathrm{mmol}$, 0.1 equiv) at $\mathrm{rt}$ according to the general procedure described above followed by flash 
chromatography over silica eluting with EtOAc/hexane (1: 10) afforded 4d $(0.078$ g, $74 \%)$ as a mixture of inseparable diastereomers ( $\mathrm{dr} 21: 1)$ as a yellow oil.

Analytical data for 4d: $\mathrm{R}_{f}=0.58($ EtOAc/hexane $1: 3) ;{ }^{1} \mathrm{H}$ NMR $\left(400 \mathrm{MHz}, \mathrm{CDCl}_{3}\right) \square$ $7.97(\mathrm{~d}, J=8.4 \mathrm{~Hz}, 2 \mathrm{H}), 7.45(\mathrm{~d}, J=8.4 \mathrm{~Hz}, 0.08 \mathrm{H}), 7.36(\mathrm{~d}, J=8.4 \mathrm{~Hz}, 1.92 \mathrm{H}), 6.73$ $(\mathrm{d}, J=8.8 \mathrm{~Hz}, 2 \mathrm{H}), 6.54(\mathrm{~d}, J=8.8 \mathrm{~Hz}, 2 \mathrm{H}), 5.5(\mathrm{~s}, 0.04 \mathrm{H}), 5.43(\mathrm{~s}, 0.96 \mathrm{H}), 5.29(\mathrm{~s}, 1$ H), $3.92(\mathrm{~d}, J=2.8 \mathrm{~Hz}, 1 \mathrm{H}), 3.91(\mathrm{~s}, 4 \mathrm{H}), 3.86-3.77(\mathrm{~m}, 1 \mathrm{H}), 3.72(\mathrm{~s}, 3 \mathrm{H}) .3 .73-3.61$ (m, $1 \mathrm{H}), 3.13(\mathrm{dd}, J=14.4 \mathrm{~Hz}, 6.0 \mathrm{~Hz}, 0.96 \mathrm{H}), 3.08-3.03(\mathrm{~m}, 0.04 \mathrm{H}), 1.71-1.64(\mathrm{~m}, 2$ H), 1.50-1.43 (m, $1 \mathrm{H}), 1.37-1.26(\mathrm{~m}, 7 \mathrm{H}), 1.14(\mathrm{t}, J=7.2 \mathrm{~Hz}, 0.12 \mathrm{H}), 1.05(\mathrm{t}, J=7.2$ $\mathrm{Hz}, 2.88 \mathrm{H}), 0.87(\mathrm{t}, J=6.8 \mathrm{~Hz}, 3 \mathrm{H}) ;{ }^{13} \mathrm{C} \mathrm{NMR}\left(125 \mathrm{MHz}, \mathrm{CDCl}_{3}\right) \square 173.2,166.8$ (166.9), 152.8, 147.5 (147.9), 147.1 (147.2), 140.9, 129.5 (129.6) two carbons, 129.0, 127.2 (126.7) two carbons, 117.3, 115.5 two carbons, 114.8 two carbons, 60.8 (62.4), 60.7 (60.9), 55.6, 52.1, $47.2(46.9), 31.7,31.0,29.3,27.3,22.6,14.0,13.9$, signals for the minor diastereomer are given in parentheses; IR (neat, $\left.\mathrm{cm}^{-1}\right) 3373(\mathrm{w}$ br), 1724 (s), 1606 (m); HRMS (ES $)$ calcd for $\mathrm{C}_{28} \mathrm{H}_{38} \mathrm{NO}_{5}\left(\mathrm{M}+\mathrm{H}^{+}\right), 468.2750$, found 468.2751.

\section{$N$-p-methoxyphenyl-2-(1-hexyl)-3-(p-methoxycarbonylphenyl)-1-(p-}

methoxycarbonylphenyl)-3-butenamine ( 5 ). Treatment of $p$ methoxycarbonylphenylboronic acid $(0.081 \mathrm{~g}, 0.450 \mathrm{mmol}, 2.0$ equiv), CsF (0.137 g, 0.900 mmol, 4.0 equiv), $\quad N$-(p-methoxyphenyl)imine of $p$ methoxycarbonylphenylcarboxaldehyde $(0.061 \mathrm{~g}, 0.225 \mathrm{mmol}, 1.0$ equiv) and 1,2nonadiene $\left(0.140 \mathrm{~g}, 1.13 \mathrm{mmol}, 5.0\right.$ equiv) with $\mathrm{Pd}(\mathrm{OAc})_{2}(0.005 \mathrm{~g}, 0.023 \mathrm{mmol}, 0.1$ equiv) and $\mathrm{H}\left[\mathrm{P}(t-\mathrm{Bu})_{3}\right] \mathrm{BF}_{4}(0.007 \mathrm{~g}, 0.023 \mathrm{mmol}, 0.1$ equiv $)$ at $40{ }^{\circ} \mathrm{C}$ according to the general procedure described above followed by flash chromatography over silica eluting 
with EtOAc/hexane (1 : 10) afforded a single diastereomer $5(0.081 \mathrm{~g}, 67 \%)$ as a colorless oil.

Analytical data for 5: $\mathrm{R}_{f}=0.59($ EtOAc/hexane $1: 2) ;{ }^{1} \mathrm{H}$ NMR $\left(400 \mathrm{MHz}, \mathrm{CDCl}_{3}\right) \square$ $7.92(\mathrm{t}, J=8.4 \mathrm{~Hz}, 4 \mathrm{H}), 7.38(\mathrm{~d}, J=8.0 \mathrm{~Hz}, 2 \mathrm{H}), 7.29(\mathrm{~d}, J=8.0 \mathrm{~Hz}, 2 \mathrm{H}), 6.61(\mathrm{~d}, J=$ $8.0 \mathrm{~Hz}, 2 \mathrm{H}), 6.32(\mathrm{~s} \mathrm{br}, 2 \mathrm{H}), 5.44(\mathrm{~s}, 1 \mathrm{H}), 5.28(\mathrm{~s}, 1 \mathrm{H}), 4.07(\mathrm{~d} \mathrm{br}, J=11.6 \mathrm{~Hz}, 2 \mathrm{H})$, 3.91 (s, 3 H), 3.88 (s, 3 H), 3.66 (s br, 3 H), 2.82 (s br, 1 H), 1.32-1.25 (m, 2 H), 1.22$1.11(\mathrm{~m}, 8 \mathrm{H}), 0.83(\mathrm{t}, J=6.8 \mathrm{~Hz}, 3 \mathrm{H}) ;{ }^{13} \mathrm{C} \mathrm{NMR}\left(125 \mathrm{MHz}, \mathrm{CDCl}_{3}\right) \square$ 166.9, 166.8, 152.2, 148.9, 148.4, 146.6 two carbons, 129.6 four carbons, 129.1 two carbons, 127.7 two carbons, 127.2 four carbons, $117.9,114.7,61.9,55.6,52.8,52.1,52.0,31.6,30.3,29.7$, 29.1, 27.3, 22.5, 14.0; IR (neat, $\left.\mathrm{cm}^{-1}\right) 3400\left(\mathrm{w}\right.$ br), 1722 (s), 1608 (w); HRMS (ES ${ }^{+}$) calcd for $\mathrm{C}_{33} \mathrm{H}_{40} \mathrm{NO}_{5}\left(\mathrm{M}+\mathrm{H}^{+}\right)$, 530.2906, found 530.2914.

\section{$N$-p-methoxyphenyl-2-(1-hexyl)-3-(p-methoxycarbonylphenyl)-1-(phenyl)-3-}

butenamine (6). Treatment of $p$-methoxycarbonylphenylboronic acid $(0.081 \mathrm{~g}, 0.450$ mmol, 2.0 equiv), $\mathrm{CsF}(0.137 \mathrm{~g}, 0.900 \mathrm{mmol}, 4.0$ equiv), $N$-( $p$-methoxyphenyl)imine of phenylcarboxaldehyde ( $0.048 \mathrm{~g}, 0.225 \mathrm{mmol}, 1.0$ equiv) and 1,2-nonadiene $(0.140 \mathrm{~g}, 1.13$ mmol, 5.0 equiv) with $\mathrm{Pd}(\mathrm{OAc})_{2}(0.005 \mathrm{~g}, 0.023 \mathrm{mmol}, 0.1$ equiv $)$ and $\mathrm{H}\left[\mathrm{P}(t-\mathrm{Bu})_{3}\right] \mathrm{BF}_{4}$ ( $0.007 \mathrm{~g}, 0.023 \mathrm{mmol}, 0.1$ equiv) at $40{ }^{\circ} \mathrm{C}$ according to the general procedure described above followed by flash chromatography over silica with a gradient elution using EtOAc/hexane $(1: 15)$ to EtOAc/hexane $(1: 10)$ afforded a single diastereomer $6(0.065$ g, 61\%) as a colorless oil.

Analytical data for 6: $\mathrm{R}_{f}=0.47($ EtOAc/hexane $1: 5) ;{ }^{1} \mathrm{H}$ NMR $\left(400 \mathrm{MHz}, \mathrm{CDCl}_{3}\right) \square$ $7.94(\mathrm{~d}, J=8.4,2 \mathrm{H}), 7.31-7.29(\mathrm{~m}, 4 \mathrm{H}), 7.25(\mathrm{t}, J=7.6 \mathrm{~Hz}, 2 \mathrm{H}), 7.17(\mathrm{t}, J=7.2 \mathrm{~Hz}, 1$ H), $6.62(\mathrm{~d}, J=8.8 \mathrm{~Hz}, 2 \mathrm{H}), 6.39(\mathrm{~d}$ br, $2 \mathrm{H}), 5.43(\mathrm{~s}, 1 \mathrm{H}), 5.28(\mathrm{~s}, 1 \mathrm{H}), 4.07(\mathrm{~d} \mathrm{br}, J=$ 
8.4 Hz, $1 \mathrm{H}), 4.06$ (s br, $1 \mathrm{H}), 3.91$ (s, $3 \mathrm{H}), 3.66$ (s, $3 \mathrm{H}), 2.82$ (s br, $1 \mathrm{H}), 1.38-1.23$ (m, 2 $\mathrm{H}), 1.23-1.07(\mathrm{~m}, 8 \mathrm{H}), 0.81(\mathrm{t}, J=6.8 \mathrm{~Hz}, 3 \mathrm{H}) ;{ }^{13} \mathrm{C} \mathrm{NMR}\left(125 \mathrm{MHz}, \mathrm{CDCl}_{3}\right) \square 166.9$, $152.0,149.5,146.9,142.8,141.9,129.5$ four carbons, 128.9, 128.3 two carbons, 127.5, 127.2 four carbons, 127.1, 117.6, 114.6, 62.0, 55.6, 53.0, 52.1, 31.6, 30.6, 29.1, 27.3, 22.5, 14.0; IR (neat, $\mathrm{cm}^{-1}$ ) 3402 (w br), 1722 (s), 1606 (m); HRMS (ES ${ }^{+}$) calcd for $\mathrm{C}_{31} \mathrm{H}_{38} \mathrm{NO}_{3}\left(\mathrm{M}+\mathrm{H}^{+}\right)$, 472.2852, found 472.2856.

\section{$N$-p-methoxyphenyl-2-(1-hexyl)-3-(p-methoxycarbonylphenyl)-1-(p-nitrophenyl)-3-}

butenamine (7). Treatment of $p$-methoxycarbonylphenylboronic acid $(0.081 \mathrm{~g}, 0.450$ mmol, 2.0 equiv), $\mathrm{CsF}(0.137 \mathrm{~g}, 0.900 \mathrm{mmol}, 4.0$ equiv), $N$-( $p$-methoxyphenyl)imine of p-nitrophenylcarboxaldehyde ( $0.058 \mathrm{~g}, 0.225 \mathrm{mmol}, 1.0$ equiv) and 1,2-nonadiene $(0.140$ $\mathrm{g}, 1.13 \mathrm{mmol}, 5.0$ equiv) with $\mathrm{Pd}(\mathrm{OAc})_{2}(0.005 \mathrm{~g}, 0.023 \mathrm{mmol}, 0.1$ equiv $)$ and $\mathrm{H}[\mathrm{P}(t-$ $\left.\mathrm{Bu})_{3}\right]_{\mathrm{BF}_{4}}\left(0.007 \mathrm{~g}, 0.023 \mathrm{mmol}, 0.1\right.$ equiv) at $40{ }^{\circ} \mathrm{C}$ according to the general procedure described above followed by flash chromatography over silica eluting with EtOAc/hexane (1 : 10) afforded a single diastereomer $7(0.073 \mathrm{~g}, 62 \%)$ as a yellow oil.

Analytical data for 7: $\mathrm{R}_{f}=0.48($ EtOAc/hexane $1: 3) ;{ }^{1} \mathrm{H}$ NMR $\left(400 \mathrm{MHz}, \mathrm{CDCl}_{3}\right) \square$ $8.09(\mathrm{~d}, J=8.8 \mathrm{~Hz}, 2 \mathrm{H}), 7.92(\mathrm{~d}, J=8.4 \mathrm{~Hz}, 2 \mathrm{H}), 7.46(\mathrm{~d}, J=8.8 \mathrm{~Hz}, 2 \mathrm{H}), 7.27(\mathrm{~d}, J=$ $9.0 \mathrm{~Hz}, 2 \mathrm{H}), 6.63$ (d, J = 8.4 Hz, $2 \mathrm{H}), 6.32$ (d br, $2 \mathrm{H}), 5.47$ (s, $1 \mathrm{H}), 5.30$ (s, $1 \mathrm{H}), 4.01$ (s br, $2 \mathrm{H}), 3.92$ (s, $3 \mathrm{H}), 3.66$ (s, $3 \mathrm{H}), 2.89$ (s br, $1 \mathrm{H}), 1.44-1.28$ (m, $2 \mathrm{H}), 1.26-1.08$ (m, $8 \mathrm{H}), 0.82(\mathrm{t}, J=6.8 \mathrm{~Hz}, 3 \mathrm{H}),{ }^{13} \mathrm{C} \mathrm{NMR}\left(125 \mathrm{MHz}, \mathrm{CDCl}_{3}\right) \square 166.7,152.4,150.8$, $148.5,147.0,146.2,140.4,129.6$ four carbons, $129.2,128.4$ two carbons, 127.1 two carbons, 123.5 two carbons, 118.2, 114.7 two carbons, 61.4, 55.6, 52.6, 52.2, 31.6, 30.5, 29.1, 27.3, 22.5, 13.9; IR (neat, $\mathrm{cm}^{-1}$ ) 3402 (w br), 1720 (s), 1606 (m), 1514 (s), 1346 (s); HRMS $\left(\mathrm{ES}^{+}\right)$calcd for $\mathrm{C}_{31} \mathrm{H}_{37} \mathrm{~N}_{2} \mathrm{O}_{5}\left(\mathrm{M}+\mathrm{H}^{+}\right)$, 517.2702, found 517.2703. 


\section{$N$-p-methoxyphenyl-2-(1-hexyl)-3-(p-methoxycarbonylphenyl)-1-(m-}

\section{nitrocarbonylphenyl)-3-butenamine (8).}

Treatment of $p$-methoxycarbonylphenylboronic acid (0.081 g, $0.450 \mathrm{mmol}, 2.0$ equiv), CsF $(0.137 \mathrm{~g}, 0.900 \mathrm{mmol}, 4.0$ equiv $), \quad N$-(p-methoxyphenyl)imine of $m$ nitrophenylcarboxaldehyde ( $0.058 \mathrm{~g}, 0.225 \mathrm{mmol}, 1.0$ equiv) and 1,2-nonadiene $(0.140 \mathrm{~g}$, $1.13 \mathrm{mmol}, 5.0$ equiv) with $\mathrm{Pd}(\mathrm{OAc})_{2}(0.005 \mathrm{~g}, 0.023 \mathrm{mmol}, 0.1$ equiv $)$ and $\mathrm{H}[\mathrm{P}(t$ $\left.\mathrm{Bu})_{3}\right]_{\mathrm{BF}_{4}}\left(0.007 \mathrm{~g}, 0.023 \mathrm{mmol}, 0.1\right.$ equiv) at $40{ }^{\circ} \mathrm{C}$ according to the general procedure described above followed by flash chromatography over silica with a gradient elution using EtOAc/hexane $(1: 10)$ to EtOAc/hexane $(1: 7)$ afforded a single diastereomer 8 $(0.073 \mathrm{~g}, 62 \%)$ as a yellow oil.

Analytical data for 8: $\mathrm{R}_{f}=0.47($ EtOAc/hexane $1: 3) ;{ }^{1} \mathrm{H}$ NMR $\left(500 \mathrm{MHz}, \mathrm{CDCl}_{3}\right) \square$ $8.13(\mathrm{~s}, 1 \mathrm{H}), 8.00(\mathrm{~d}, J=8.0 \mathrm{~Hz}, 1 \mathrm{H}), 7.91(\mathrm{~d}, J=8.5 \mathrm{~Hz}, 2 \mathrm{H}), 7.60(\mathrm{~d}, J=7.5 \mathrm{~Hz}, 1$ H), $7.38(\mathrm{t}, J=8.0 \mathrm{~Hz}, 1 \mathrm{H}), 7.26(\mathrm{~d}, J=8.0 \mathrm{~Hz}, 2 \mathrm{H}), 6.63(\mathrm{~d}, J=8.0 \mathrm{~Hz}, 2 \mathrm{H}), 6.34(\mathrm{~d}$ br, $2 \mathrm{H}), 5.48$ (s, $1 \mathrm{H}), 5.30$ (s, $1 \mathrm{H}), 4.22$ (d br, J = 5. Hz, $2 \mathrm{H}), 3.91$ (s, $3 \mathrm{H}), 3.67$ (s br, 3 H), 2.89 (s br, $1 \mathrm{H}), 1.60-1.24(\mathrm{~m}, 2 \mathrm{H}), 1.22-1.15(\mathrm{~m}, 8 \mathrm{H}), 0.83(\mathrm{t}, J=7.0 \mathrm{~Hz}, 3 \mathrm{H}) ;{ }^{13} \mathrm{C}$ NMR (125 MHz, $\left.\mathrm{CDCl}_{3}\right) \square 166.6,152.3,148.3,148.2,146.4,145.2,140.4,133.6,129.6$ two carbons, 129.1, 129.0 two carbons, 127.0 two carbons, 122.3, 122.2 two carbons, $118.1,114.7,114.5,61.0,55.6,52.5,52.1,31.5,30.6,29.1,27.3,22.5,13.9 ;$ IR (neat, $\mathrm{cm}^{-}$

$\left.{ }^{1}\right) 3402\left(\mathrm{w}\right.$ br), 1720 (s), $1606(\mathrm{w}), 1529$ (s), $1512(\mathrm{~s})$; HRMS (ES ${ }^{+}$) calcd for $\mathrm{C}_{31} \mathrm{H}_{37} \mathrm{~N}_{2} \mathrm{O}_{5}$ $\left(\mathrm{M}+\mathrm{H}^{+}\right)$, 517.2702, found 517.2709. 
$N$-p-methoxyphenyl-2-(1-hexyl)-3-(p-methoxycarbonylphenyl)-1-(p-methoxyphenyl)-

\section{3-butenamine (9).}

Treatment of $p$-methoxycarbonylphenylboronic acid $(0.081 \mathrm{~g}, 0.450 \mathrm{mmol}, 2.0$ equiv), CsF (0.137 g, $0.900 \mathrm{mmol}, 4.0$ equiv), $N$-( $p$-methoxyphenyl)imine of $p$ methoxyphenylcarboxaldehyde (0.054 g, $0.225 \mathrm{mmol}, 1.0$ equiv) and 1,2-nonadiene $\left(0.140 \mathrm{~g}, 1.13 \mathrm{mmol}, 5.0\right.$ equiv) with $\mathrm{Pd}(\mathrm{OAc})_{2}(0.005 \mathrm{~g}, 0.023 \mathrm{mmol}, 0.1$ equiv) and $\mathrm{H}\left[\mathrm{P}(t-\mathrm{Bu})_{3}\right] \mathrm{BF}_{4}(0.007 \mathrm{~g}, 0.023 \mathrm{mmol}, 0.1$ equiv $)$ at $\mathrm{rt}$ according to the general procedure described above followed by flash chromatography over silica eluting with EtOAc/hexane (1 : 10) afforded $9(0.053 \mathrm{~g}, 47 \%)$ as a mixture of inseparable diastereomers (dr $8: 1)$ as a yellow oil. An additional purification afforded a sample containing only traces of the minor isomer (see data below).

Analytical data for 9: $\mathrm{R}_{f}=0.53($ EtOAc/hexane $1: 3) ;{ }^{1} \mathrm{H}$ NMR $\left(400 \mathrm{MHz}, \mathrm{CDCl}_{3}\right) \square$ $7.94(\mathrm{~d}, J=8.4 \mathrm{~Hz}, 2 \mathrm{H}), 7.31(\mathrm{~d}, J=8.4 \mathrm{~Hz}, 2 \mathrm{H}), 7.21(\mathrm{~d}, J=8.4 \mathrm{~Hz}, 2 \mathrm{H}), 6.79(\mathrm{~d}, J=$ $8.8 \mathrm{~Hz}, 2 \mathrm{H}), 6.62(\mathrm{~d}, J=8.4 \mathrm{~Hz}, 2 \mathrm{H}), 6.38(\mathrm{~d} \mathrm{br}, 2 \mathrm{H}), 5.42(\mathrm{~s}, 1 \mathrm{H}), 5.27(\mathrm{~s}, 1 \mathrm{H}), 4.02$ (s br, $1 \mathrm{H}), 3.91$ (s, $3 \mathrm{H}), 3.76$ (s, $3 \mathrm{H}), 3.66$ (s br, $3 \mathrm{H}), 2.77$ (s br, $1 \mathrm{H}), 1.39-1.24$ (m, 2 $\mathrm{H}), 1.23-1.16(\mathrm{~m}, 3 \mathrm{H}), 1.15-1.11(\mathrm{~m}, 5 \mathrm{H}), 0.82(\mathrm{t}, J=6.8 \mathrm{~Hz}, 3 \mathrm{H}) ;{ }^{13} \mathrm{C}$ NMR $(125$ $\left.\mathrm{MHz}, \mathrm{CDCl}_{3}\right) \square 166.9,158.6$ (157.9), 151.9, 149.6, 146.9, 141.5, 134.7, 129.5 four carbons, $128.9,128.5$ two carbons, 127.2 two carbons, 117.5, 114.3 two carbons, 113.7 two carbons, $61.5,55.6,55.2,53.3,52.1,31.6,30.5,29.0,27.3,22.6,14.0$, signals for the minor diastereomer are given in parentheses; IR (neat, $\mathrm{cm}^{-1}$ ) 3406 (s br), 1720 (s), 1608 (m), $1512(\mathrm{~s})$; HRMS $\left(\mathrm{ES}^{+}\right)$calcd for $\mathrm{C}_{32} \mathrm{H}_{40} \mathrm{NO}_{4}\left(\mathrm{M}+\mathrm{H}^{+}\right)$, 502.2957, found 502.2950. 
( \pm$)-(1 R, 2 R)-N$-p-methoxyphenyl-2-(1-hexyl)-3-(p-methoxycarbonylphenyl)-1-(3pyridyl)-3-butenamine (10).

Treatment of $p$-methoxycarbonylphenylboronic acid $(0.081 \mathrm{~g}, 0.450 \mathrm{mmol}, 2.0$ equiv), CsF (0.137 g, $0.900 \mathrm{mmol}, 4.0$ equiv), $N$-(p-methoxyphenyl)imine of 3 pyridylcarboxaldehyde ( $0.048 \mathrm{~g}, 0.225 \mathrm{mmol}, 1.0$ equiv) and 1,2-nonadiene $(0.140 \mathrm{~g}$, $1.13 \mathrm{mmol}, 5.0$ equiv) with $\mathrm{Pd}(\mathrm{OAc})_{2}(0.005 \mathrm{~g}, 0.023 \mathrm{mmol}, 0.1$ equiv $)$ and $\mathrm{H}[\mathrm{P}(t-$ $\left.\mathrm{Bu})_{3}\right]_{\mathrm{BF}_{4}}\left(0.007 \mathrm{~g}, 0.023 \mathrm{mmol}, 0.1\right.$ equiv) at $40{ }^{\circ} \mathrm{C}$ according to the general procedure described above followed by flash chromatography over silica with a gradient elution using EtOAc/hexane $(1: 3)$ to EtOAc/hexane $(1: 2)$ afforded a single diastereomer 10 $(0.060 \mathrm{~g}, 57 \%)$ as a white solid.

Analytical data for 10: $\mathrm{mp} 91-93{ }^{\circ} \mathrm{C} ; \mathrm{R}_{f}=0.49($ EtOAc/hexane $1: 1) ;{ }^{1} \mathrm{H}$ NMR (400 $\left.\mathrm{MHz}, \mathrm{CDCl}_{3}\right) \square 8.54(\mathrm{~s}, 1 \mathrm{H}), 8.41(\mathrm{~d}, J=3.2 \mathrm{~Hz}, 1 \mathrm{H}), 7.93(\mathrm{~d}, J=8.4 \mathrm{~Hz}, 2 \mathrm{H}), 7.61(\mathrm{~d}$, $J=8.0 \mathrm{~Hz}, 1 \mathrm{H}), 7.28(\mathrm{~d}, J=8.4 \mathrm{~Hz}, 2 \mathrm{H}), 7.16(\mathrm{dd}, J=7.6 \mathrm{~Hz}, 5.2 \mathrm{~Hz}, 1 \mathrm{H}), 6.63(\mathrm{~d}, J=$ $6.8 \mathrm{~Hz}, 2 \mathrm{H}), 6.35(\mathrm{~d}, J=12.4 \mathrm{~Hz}, 2 \mathrm{H}), 5.46(\mathrm{~s}, 1 \mathrm{H}), 5.28(\mathrm{~s}, 1 \mathrm{H}), 4.13(\mathrm{~d}, J=6.0 \mathrm{~Hz}, 1$ H), 4.11 (s br, $1 \mathrm{H}), 3.91(\mathrm{~s}, 3 \mathrm{H}), 3.67(\mathrm{~s}, 3 \mathrm{H}), 2.86$ (dt, $J=8.0 \mathrm{~Hz}, 4.4 \mathrm{~Hz}, 1 \mathrm{H}), 1.43-$ $1.32(\mathrm{~m}, 3 \mathrm{H}), 1.27-1.09(\mathrm{~m}, 7 \mathrm{H}), 0.82(\mathrm{t}, J=6.8 \mathrm{~Hz}, 3 \mathrm{H}) ;{ }^{13} \mathrm{C} \mathrm{NMR}\left(125 \mathrm{MHz}, \mathrm{CDCl}_{3}\right)$ $\square$ 166.7, 152.3, 149.4, 148.6, 148.3, 146.5, 140.6, 138.1, 134.9, 129.6 two carbons, 129.2, 127.1 two carbons, $123.4,117.9,114.7$ two carbons, 114. 6 two carbons, 59.5, 55.6, 52.7, 52.1 31.6, 30.4, 29.1, 27.3, 22.5, 13.9; IR (neat, $\mathrm{cm}^{-1}$ ) 3398 (w br), 1720 (s), $1606(\mathrm{~m})$; HRMS $\left(\mathrm{ES}^{+}\right)$calcd for $\mathrm{C}_{30} \mathrm{H}_{37} \mathrm{~N}_{2} \mathrm{O}_{3}\left(\mathrm{M}+\mathrm{NH}_{4}^{+}\right)$, 473.2804, found 473.2805. 


\section{$N$-p-methoxyphenyl-2-(1-hexyl)-3-(p-methoxycarbonylphenyl)-1-(cyclohexyl)-3-}

\section{butenamine (11).}

Treatment of $p$-methoxycarbonylphenylboronic acid ( $0.081 \mathrm{~g}, 0.450 \mathrm{mmol}, 2.0$ equiv),

CsF $(0.137$ g, 0.900 mmol, 4.0 equiv $), N$-( $p$-methoxyphenyl)imine of cyclohexylcarboxaldehyde ( $0.049 \mathrm{~g}, 0.225 \mathrm{mmol}, 1.0$ equiv) and 1,2-nonadiene ( $0.140 \mathrm{~g}$, 1.13 mmol, 5.0 equiv) with $\mathrm{Pd}(\mathrm{OAc})_{2}(0.005 \mathrm{~g}, 0.023 \mathrm{mmol}, 0.1$ equiv $)$ and $\mathrm{H}[\mathrm{P}(t-$ $\left.\mathrm{Bu})_{3}\right] \mathrm{BF}_{4}\left(0.007 \mathrm{~g}, 0.023 \mathrm{mmol}, 0.1\right.$ equiv) at $40{ }^{\circ} \mathrm{C}$ according to the general procedure described above followed by flash chromatography over silica using EtOAc/hexane (1 : 20) afforded a single diastereomer $11(0.069 \mathrm{~g}, 64 \%)$ as a colorless oil.

Analytical data for 11: $\mathrm{R}_{f}=0.46(\mathrm{EtOAc} / \mathrm{hexane} 1: 5) ;{ }^{1} \mathrm{H}$ NMR $\left(400 \mathrm{MHz}, \mathrm{CDCl}_{3}\right) \square$ $7.96(\mathrm{~d}, J=8.4 \mathrm{~Hz}, 2 \mathrm{H}), 7.31(\mathrm{~d}, J=8.4 \mathrm{~Hz}, 2 \mathrm{H}), 6.69(\mathrm{~d}, J=8.8 \mathrm{~Hz}, 2 \mathrm{H}), 6.39(\mathrm{~d}, J=$ $8.4 \mathrm{~Hz}, 2 \mathrm{H}), 5.36$ (s, $1 \mathrm{H}), 5.19$ (s, $1 \mathrm{H}), 3.92$ (s, $3 \mathrm{H}), 3.73$ (s, $3 \mathrm{H}), 3.26$ (s br, $1 \mathrm{H}), 3.14$ (t, $J=5.6 \mathrm{~Hz}, 1 \mathrm{H}), 2.95(\mathrm{dt}, J=8.4 \mathrm{~Hz}, 5.2 \mathrm{~Hz}, 1 \mathrm{H}), 1.74(\mathrm{~d} \mathrm{br}, J=11.6 \mathrm{~Hz}, 1 \mathrm{H}), 1.70-$ $1.52(\mathrm{~m}, 2 \mathrm{H}), 1.51-1.38(\mathrm{~m}, 4 \mathrm{H}), 1.35-1.11(\mathrm{~m}, 9 \mathrm{H}), 1.05-0.73(\mathrm{~m}, 5 \mathrm{H}), 0.87(\mathrm{t}, J=6.4$ $\mathrm{Hz}, 3 \mathrm{H}) ;{ }^{13} \mathrm{C}$ NMR $\left(125 \mathrm{MHz}, \mathrm{CDCl}_{3}\right) \square 166.9,150.8,150.0,149.5,143.9,129.5$ four carbons, 128.6, 126.7 two carbons, 116.2, 114.8, 113.2, 62.2, 55.8, 52.0, 46.5, 41.9, 32.9, $31.8,31.6,29.5,28.8,27.8,26.3,26.2,26.1,22.6,14.1$; IR (neat, $\mathrm{cm}^{-1}$ ) 3400 (w br), 1724 (w), 1606 (m); HRMS (ES ${ }^{+}$) calcd for $\mathrm{C}_{31} \mathrm{H}_{44} \mathrm{NO}_{3}\left(\mathrm{M}+\mathrm{H}^{+}\right), 478.3321$, found 478.3318 . 


\section{$N$-p-methoxyphenyl-2-(1-hexyl)-3-(trans-2-phenyl-1-ethenyl)-1-(p-}

\section{methoxycarbonylphenyl)-3-butenamine (12).}

Treatment of trans-2-phenylvinylboronic acid (0.067 g, $0.450 \mathrm{mmol}, 2.0$ equiv), CsF $(0.137 \mathrm{~g}, \quad 0.900 \mathrm{mmol}, \quad 4.0$ equiv), $\quad N$-( $p$-methoxyphenyl)imine of $p$ methoxycarbonylphenylcarboxaldehyde $(0.061 \mathrm{~g}, 0.225 \mathrm{mmol}, 1.0$ equiv) and 1,2nonadiene $\left(0.140 \mathrm{~g}, 1.13 \mathrm{mmol}, 5.0\right.$ equiv) with $\mathrm{Pd}(\mathrm{OAc})_{2}(0.005 \mathrm{~g}, 0.023 \mathrm{mmol}, 0.1$ equiv) and $\mathrm{H}\left[\mathrm{P}(t-\mathrm{Bu})_{3}\right] \mathrm{BF}_{4}(0.007 \mathrm{~g}, 0.023 \mathrm{mmol}, 0.1$ equiv $)$ at $40{ }^{\circ} \mathrm{C}$ according to the general procedure described above followed by flash chromatography over silica using EtOAc/hexane (1 : 15) afforded $12(0.077 \mathrm{~g}, 68 \%)$ as a mixture of inseparable diastereomers $(\mathrm{dr} 7: 1)$ as a yellow oil.

Analytical data for 12: $\mathrm{R}_{f}=0.45(\mathrm{EtOAc} / \mathrm{hexane} 1: 5) ;{ }^{1} \mathrm{H} \mathrm{NMR}\left(400 \mathrm{MHz}, \mathrm{CD}_{3} \mathrm{CN}\right) \square$ $7.91(\mathrm{~d}, J=8.4 \mathrm{~Hz}, 1.75 \mathrm{H}), 7.80(\mathrm{~d}, J=8.4 \mathrm{~Hz}, 0.25 \mathrm{H}), 7.53(\mathrm{~d}, J=8.4 \mathrm{~Hz}, 2 \mathrm{H}), 7.40$ (d, $J=7.2 \mathrm{~Hz}, 2 \mathrm{H}), 7.30(\mathrm{t}, J=7.2 \mathrm{~Hz}, 2 \mathrm{H}), 7.23(\mathrm{t}, J=4.0 \mathrm{~Hz}, 1 \mathrm{H}), 6.84(\mathrm{~d}, J=16.4$ $\mathrm{Hz}, 1 \mathrm{H}), 6.72(\mathrm{~d}, J=16.4 \mathrm{~Hz}, 1 \mathrm{H}), 6.57(\mathrm{~d}, J=8.8 \mathrm{~Hz}, 2 \mathrm{H}), 6.43(\mathrm{~d}, J=9.2 \mathrm{~Hz}, 2 \mathrm{H})$, $5.44(\mathrm{~s}, 0.87 \mathrm{H}), 5.26(\mathrm{~s}, 0.13 \mathrm{H}), 5.17(\mathrm{~s}, 0.87 \mathrm{H}), 5.07$ (s, $0.13 \mathrm{H}), 4.48$ (s br, $1 \mathrm{H}), 4.43$ (d, $J=8.8 \mathrm{~Hz}, 1 \mathrm{H}), 3.81(\mathrm{~s}, 2.63 \mathrm{H}), 3.77(\mathrm{~s}, 0.37 \mathrm{H}), 3.59(\mathrm{~s}, 0.37 \mathrm{H}), 3.57(\mathrm{~s}, 2.63 \mathrm{H})$, 2.90 (ddd, $J=10.8 \mathrm{~Hz}, 8.0 \mathrm{~Hz}, 2.8 \mathrm{~Hz}, 0.13 \mathrm{H}$ ), 2.80-2.76 (m, 0.87 H), 1.49-1.38 (m, 1 $\mathrm{H}), 1.29-1.08(\mathrm{~m}, 9 \mathrm{H}), 0.78(\mathrm{t}, J=6.8 \mathrm{~Hz}, 3 \mathrm{H})$;

${ }^{13} \mathrm{C}$ NMR (125 MHz, $\left.\mathrm{CD}_{3} \mathrm{CN}\right) \square 168.1,153.3$ (153.2), 151.2 (150.8), 148.4 (148.1), 143.3 (143.5), 138.9 (138.8), 132.2, 131.4, 130.7 two carbons, 130.6, 130.3, 130.2 two carbons, 129.6 two carbons, 129.2 two carbons, (118.2) 117.3, 115.9 (116.0) two carbons, 115.8 two carbons, 62.9 (63.3), 56.6 (56.7), 53.1 (53.2), 50.5 (49.4), 32.9 (33.0), 32.5 (31.7), 30.4 (30.6), 28.4 (28.6), 23.8 (23.9), 14.8 (14.9) signals for the minor diastereomer are 
given in parentheses; IR (neat, $\left.\mathrm{cm}^{-1}\right) 3398\left(\mathrm{w}\right.$ br), 1720 (s), $1610(\mathrm{w})$; HRMS $\left(\mathrm{ES}^{+}\right)$calcd for $\mathrm{C}_{33} \mathrm{H}_{40} \mathrm{NO}_{3}\left(\mathrm{M}+\mathrm{H}^{+}\right)$, 498.3008, found 498.3006.

\section{Ethyl-3-(1-hexyl)-4-(p-methoxyphenyl)-2-( $N$-p-methoxyphenyl- $N$-allylamino)-4- pentenoate (13a).}

Compound 13a was prepared according to a modified literature procedure. ${ }^{4 a, b}$ A solution of a $10: 1$ mixture of diastereomers of amine $4 \mathbf{c}(0.074 \mathrm{~g}, 0.169 \mathrm{mmol}, 1.0$ equiv) in dry $\mathrm{CH}_{3} \mathrm{CN}(1.7 \mathrm{~mL})$ was injected into a flask containing $\mathrm{K}_{2} \mathrm{CO}_{3}(0.070 \mathrm{~g}, 0.508 \mathrm{mmol}, 3.0$ equiv) and $\mathrm{NaI}$ (0.003 g, $0.017 \mathrm{mmol}, 0.1$ equiv). Allyl bromide $(0.123 \mathrm{~g}, 1.02 \mathrm{mmol}$, $85.9 \square \mathrm{L}, 6.0$ equiv) was then injected and the solution was stirred at $80{ }^{\circ} \mathrm{C}$ for $23 \mathrm{~h}$. Water $(20 \mathrm{~mL})$ was added, and the mixture was extracted with ether $(3 \times 20 \mathrm{~mL})$. Organic extracts were dried $\left(\mathrm{MgSO}_{4}\right)$, and the solvents were removed under reduced pressure to afford the crude product. Separation by flash chromatography over silica eluting with EtOAc/Hexane $(1: 20)$ afforded a single diastereomer amine 13a $(0.064 \mathrm{~g}, 78 \%)$ as a pale yellow oil.

Analytical data for 13a. $\mathrm{R}_{f}=0.54($ EtOAc/hexane $1: 5) ;{ }^{1} \mathrm{H} \mathrm{NMR}\left(400 \mathrm{MHz}, \mathrm{CDCl}_{3}\right)$ $7.17(\mathrm{~d}, J=9.2 \mathrm{~Hz}, 2 \mathrm{H}), 6.81(\mathrm{~d}, J=11.6 \mathrm{~Hz}, 2 \mathrm{H}), 6.71(\mathrm{~d}, J=9.2 \mathrm{~Hz}, 2 \mathrm{H}), 6.66(\mathrm{~d}, J$ $=9.2 \mathrm{~Hz}, 2 \mathrm{H}), 5.41(\mathrm{oct}, J=5.2 \mathrm{~Hz}, 1 \mathrm{H}), 5.21(\mathrm{~s}, 1 \mathrm{H}), 5.02(\mathrm{~s}, 1 \mathrm{H}), 4.97(\mathrm{dd}, J=17.6$ $\mathrm{Hz}, 1.6 \mathrm{~Hz}, 1 \mathrm{H}), 4.92(\mathrm{dd}, J=10.0 \mathrm{~Hz}, 2.0 \mathrm{~Hz}, 1 \mathrm{H}), 4.14(\mathrm{~d}, J=10.8 \mathrm{~Hz}, 1 \mathrm{H}), 4.11(\mathrm{q}$, $J=7.2 \mathrm{~Hz}, 2 \mathrm{H}), 3.80(\mathrm{~s}, 3 \mathrm{H}), 3.73(\mathrm{~s}, 3 \mathrm{H}), 3.68(\mathrm{qd}, J=17.2 \mathrm{~Hz}, 6.0 \mathrm{~Hz}, 2 \mathrm{H}), 3.22(\mathrm{td}$, $J=10.4 \mathrm{~Hz}, 2.8 \mathrm{~Hz}, 1 \mathrm{H}), 1.54-1.37(\mathrm{~m}, 3 \mathrm{H}), 1.36-1.21(\mathrm{~m}, 7 \mathrm{H}), 1.19(\mathrm{t}, J=6.8 \mathrm{~Hz}, 3$ $\mathrm{H}), 0.87(\mathrm{t}, J=6.4 \mathrm{~Hz}, 3 \mathrm{H}) ;{ }^{13} \mathrm{C} \mathrm{NMR}\left(125 \mathrm{MHz}, \mathrm{CDCl}_{3}\right) \square 171.5,158.8,152.7,148.2$, 142.5, 136.0, 135.0, 128.2 two carbons, 118.4 two carbons, $115.9,114.5,113.9$ two carbons, 113.4 two carbons, 68.1, 60.3, 55.5, 55.3, 48.2, 45.1, 31.7, 31.5, 29.4, 27.0, 22.6, 
14.3, 14.1; IR (neat, $\left.\mathrm{cm}^{-1}\right) 1728(\mathrm{~s}), 1606(\mathrm{~m})$; HRMS $\left(\mathrm{ES}^{+}\right)$calcd for $\mathrm{C}_{30} \mathrm{H}_{42} \mathrm{NO}_{4}\left(\mathrm{M}+\mathrm{H}^{+}\right)$, 480.3114, found 480.3105 .

\section{$N$-p-methoxyphenyl- $N$-allyl-2-(1-hexyl)-3-(p-methoxycarbonylphenyl)-1-(phenyl)-3- butenamine (13b).}

Compound $\mathbf{1 3 b}$ was prepared according to a modified literature procedure. ${ }^{4 a, b}$ A solution of amine $6(0.044 \mathrm{~g}, 0.093 \mathrm{mmol}, 1.0$ equiv) in dry DMF (1.0 mL) was injected into a flask containing $\mathrm{K}_{2} \mathrm{CO}_{3}(0.038 \mathrm{~g}, 0.278 \mathrm{mmol}, 3.0$ equiv). Allyl bromide $(0.067 \mathrm{~g}, 0.556$ mmol, $47.0 \square \mathrm{L}, 6.0$ equiv) was then injected and the solution was stirred at $120^{\circ} \mathrm{C}$ for 9 h. Water $(20 \mathrm{~mL})$ was added, and the mixture was extracted with ether $(3 \times 20 \mathrm{~mL})$. Organic extracts were dried $\left(\mathrm{MgSO}_{4}\right)$, and the solvents were removed under reduced pressure to afford the crude product. Separation by flash chromatography over silica eluting with EtOAc/Hexane $(1: 20)$ afforded $\mathbf{1 3 b}(0.039 \mathrm{~g}, 83 \%)$ as a colorless oil.

Analytical data for 13b. $\mathrm{R}_{f}=0.45($ EtOAc/hexane $1: 7) ;{ }^{1} \mathrm{H}$ NMR $\left(400 \mathrm{MHz}, \mathrm{CDCl}_{3}\right) \square$ $7.99(\mathrm{~d}, J=8.4 \mathrm{~Hz}, 2 \mathrm{H}), 7.41(\mathrm{~d}, J=8.4 \mathrm{~Hz}, 2 \mathrm{H}), 7.25-7.199(\mathrm{~m}, 3 \mathrm{H}), 7.03(\mathrm{~d}, J=8.0$ Hz, $2 \mathrm{H}), 6.69(\mathrm{~d}, J=9.2 \mathrm{~Hz}, 2 \mathrm{H}), 6.57(\mathrm{~d}, J=8.8 \mathrm{~Hz}, 2 \mathrm{H}), 5.42(\mathrm{~s}, 1 \mathrm{H}), 5.20(\mathrm{~s}, 1 \mathrm{H})$, 5.05 (oct, $J=6.4 \mathrm{~Hz}, 1 \mathrm{H}), 4.75(\mathrm{~d}, J=9.2 \mathrm{~Hz}, 1 \mathrm{H}), 4.72(\mathrm{~s} \mathrm{br}, 1 \mathrm{H}), 4.33(\mathrm{~d}, J=9.6 \mathrm{~Hz}$, $1 \mathrm{H}), 3.93(\mathrm{~s}, 3 \mathrm{H}), 3.75(\mathrm{~s}, 3 \mathrm{H}), 3.36-3.31(\mathrm{~m}, 2 \mathrm{H}), 3.10(\mathrm{dd}, J=15.2 \mathrm{~Hz}, 7.2 \mathrm{~Hz}, 1 \mathrm{H})$, 1.57-1.48 (m, $1 \mathrm{H}), 1.47-1.32(\mathrm{~m}, 2 \mathrm{H}), 1.30-1.12(\mathrm{~m}, 7 \mathrm{H}), 0.84(\mathrm{t}, J=6.8 \mathrm{~Hz}, 3 \mathrm{H}) ;{ }^{13} \mathrm{C}$ NMR $\left(125 \mathrm{MHz}, \mathrm{CDCl}_{3}\right) \square 167.1,153.8,150.1,148.6,142.6,137.7,135.8,129.5$ two carbons, 128.6, 128.3 two carbons, 127.8 two carbons, 127.1 three carbons, 122.1 two carbons, 116.2, 115.9, 113.6 two carbons, 71.9, 55.4, 52.1, 49.8, 45.7, 31.6, 30.8, 29.4, 27.5, 22.6, 14.1; IR (neat, $\left.\mathrm{cm}^{-1}\right) 1722(\mathrm{~s})$; HRMS $\left(\mathrm{ES}^{+}\right)$calcd for $\mathrm{C}_{34} \mathrm{H}_{42} \mathrm{NO}_{3}\left(\mathrm{M}+\mathrm{H}^{+}\right)$, 512.3165 , found 512.3159 . 


\section{$N$-(p-methoxyphenyl)-1,2,3,6-tetrahydro-2-ethoxycarbonyl-3-(1-hexyl)-4-p-}

\section{methoxyphenylpyridine (14a).}

Compound 14a was prepared according to a modified literature procedure. ${ }^{5}$ Amine 13a (0.018 g, $0.038 \mathrm{mmol}, 1.0$ equiv) was dissolved in dry $\mathrm{CH}_{2} \mathrm{Cl}_{2}(3.8 \mathrm{~mL})$ and degassed by bubbling argon gas through the solution for 30 minutes. Grubb's $2^{\text {nd }}$ Generation catalyst ([1,3-bis(2,4,6-trimethylphenyl)-2-

imidazolidinylidene]dichloro(phenylmethylene)(tricyclohexylphosphine)ruthenium)

( $0.003 \mathrm{~g}, 0.004 \mathrm{mmol}, 0.1$ equiv) was then added and the solution stirred at reflux for 3.5 h. The solution was cooled to rt and DMSO (0.015 g, $0.188 \mathrm{mmol}, 13.3 \square \mathrm{L}, 5.0$ equiv) was added in order to remove the Grubb's catalyst. After stirring for an additional $24 \mathrm{~h}$, water $(20 \mathrm{~mL})$ was added, and the mixture was extracted with $\mathrm{CH}_{2} \mathrm{Cl}_{2}(4 \times 20 \mathrm{~mL})$. Organic extracts were dried $\left(\mathrm{MgSO}_{4}\right)$, and the solvents were removed under reduced pressure to afford the crude product. Separation by flash chromatography over silica eluting with EtOAc/Hexane (1 : 10) afforded tetrahydropyridine 14a $(0.017 \mathrm{~g}, 100 \%)$ as a yellow oil.

Analytical data for 14a. $\mathrm{R}_{f}=0.44($ EtOAc/hexane $1: 5) ;{ }^{1} \mathrm{H} \mathrm{NMR}\left(400 \mathrm{MHz}, \mathrm{CDCl}_{3}\right) \square$ $7.32(\mathrm{~d}, J=6.8 \mathrm{~Hz}, 2 \mathrm{H}), 6.87(\mathrm{~d}, J=10.0 \mathrm{~Hz}, 4 \mathrm{H}), 6.82(\mathrm{~d}, J=9.2 \mathrm{~Hz}, 2 \mathrm{H}), 5.91(\mathrm{t}, J=$ $3.2 \mathrm{~Hz}, 1 \mathrm{H}), 4.59(\mathrm{~d}, J=2.0 \mathrm{~Hz}, 1 \mathrm{H}), 4.07(\mathrm{q}, J=7.1 \mathrm{~Hz}, 2 \mathrm{H}), 4.03(\mathrm{q}, J=1.6 \mathrm{~Hz}, 1$ H), $3.98(\mathrm{~d}, J=3.2 \mathrm{~Hz}, 1 \mathrm{H}), 3.82(\mathrm{~s}, 3 \mathrm{H}), 3.77(\mathrm{~s}, 3 \mathrm{H}), 3.27(\mathrm{~d} \mathrm{br}, J=7.6 \mathrm{~Hz}, 1 \mathrm{H})$, 1.63-1.51 (m, $1 \mathrm{H}), 1.50-1.31(\mathrm{~m}, 1 \mathrm{H}), 1.30-1.17(\mathrm{~m}, 8 \mathrm{H}), 1.49$ (t, $J=6.8 \mathrm{~Hz}, 3 \mathrm{H}), 0.84$ $(\mathrm{t}, J=6.8 \mathrm{~Hz}, 3 \mathrm{H}) ;{ }^{13} \mathrm{C} \mathrm{NMR}\left(125 \mathrm{MHz}, \mathrm{CDCl}_{3}\right) \square 172.7,158.9,152.2,144.1,137.7$, $132.8,126.7$ two carbons, $119.9,114.7$ two carbons, 114.6 two carbons, 113.8 two carbons, $60.6,59.1,55.7,55.3,45.9,40.8,32.8,31.7,29.2,27.9,22.5,14.2,14.0$; IR 
(neat, $\left.\mathrm{cm}^{-1}\right) 1737(\mathrm{~m}), 1724(\mathrm{~m})$; HRMS $\left(\mathrm{ES}^{+}\right)$calcd for $\mathrm{C}_{28} \mathrm{H}_{38} \mathrm{NO}_{4}\left(\mathrm{M}+\mathrm{H}^{+}\right), 452.2801$, found 452.2787

\section{$N$-(p-methoxyphenyl)-1,2,3,6-tetrahydro-2-phenyl-3-(1-hexyl)-4-p- methoxycarbonylphenylpyridine (14b).}

Compound 14b was prepared according to a modified literature procedure. ${ }^{5}$ Amine $\mathbf{1 3 b}$ (0.036 g, $0.070 \mathrm{mmol}, 1.0$ equiv) was dissolved in dry $\mathrm{CH}_{2} \mathrm{Cl}_{2}(7.0 \mathrm{~mL})$ and degassed by bubbling argon gas through the solution for 30 minutes. Grubb's $2^{\text {nd }}$ Generation catalyst ([1,3-bis(2,4,6-trimethylphenyl)-2-

imidazolidinylidene]dichloro(phenylmethylene)(tricyclohexylphosphine)ruthenium)

(0.006 $\mathrm{g}, 0.007 \mathrm{mmol}, 0.1$ equiv) was then added and the solution stirred at reflux for 2.5

h. The solution was cooled to rt and DMSO (0.027 g, $0.352 \mathrm{mmol}, 25.0 \square \mathrm{L}, 5.0$ equiv) was added in order to remove the Grubb's catalyst. After stirring for an additional $24 \mathrm{~h}$, water $(20 \mathrm{~mL})$ was added, and the mixture was extracted with $\mathrm{CH}_{2} \mathrm{Cl}_{2}(4 \times 20 \mathrm{~mL})$. Organic extracts were dried $\left(\mathrm{MgSO}_{4}\right)$, and the solvents were removed under reduced pressure to afford the crude product. Separation by flash chromatography over silica eluting with EtOAc/Hexane (1 : 17) afforded tetrahydropyridine $14 \mathbf{b}(0.029 \mathrm{~g}, 84 \%)$ as a white solid.

Analytical Data for 14b. $\operatorname{mp} 103-105{ }^{\circ} \mathrm{C} ; \mathrm{R}_{f}=0.47($ EtOAc/hexane $1: 5) ;{ }^{1} \mathrm{H}$ NMR $\left(400 \mathrm{MHz}, \mathrm{CDCl}_{3}\right) \square 7.97(\mathrm{~d}, J=8.4 \mathrm{~Hz}, 2 \mathrm{H}), 7.35(\mathrm{~d}, J=8.4 \mathrm{~Hz}, 2 \mathrm{H}), 7.23-7.13(\mathrm{~m}, 3$ H), 7.12-7.10 (m, $2 \mathrm{H}), 6.85(\mathrm{~d}, J=9.2 \mathrm{~Hz}, 2 \mathrm{H}), 6.77(\mathrm{~d}, J=9.2 \mathrm{~Hz}, 2 \mathrm{H}), 6.21(\mathrm{t}, J=$ $3.6 \mathrm{~Hz}, 1 \mathrm{H}), 5.07$ (s br, $1 \mathrm{H}), 4.09$ (dd, $J=18.0 \mathrm{~Hz}, 3.2 \mathrm{~Hz}, 1 \mathrm{H}), 3.90$ (s, $3 \mathrm{H}), 3.83$ (dd, $J=18.4 \mathrm{~Hz}, 4.0 \mathrm{~Hz}, 1 \mathrm{H}), 3.76(\mathrm{~s}, 3 \mathrm{H}), 3.14(\mathrm{~d} \mathrm{br}, J=10.2 \mathrm{~Hz}, 1 \mathrm{H}), 1.78-1.70(\mathrm{~m}, 1 \mathrm{H})$, 1.57-1.42 (m, $2 \mathrm{H}), 1.39-1.15(\mathrm{~m}, 7 \mathrm{H}), 0.84(\mathrm{t}, J=6.4 \mathrm{~Hz}, 3 \mathrm{H}) ;{ }^{13} \mathrm{C}$ NMR $(125 \mathrm{MHz}$, 
$\left.\mathrm{CDCl}_{3}\right) \square 166.9,151.8,145.3,143.7,141.8,138.6,129.8$ two carbons, 128.7, 128.2 two carbons, 126.7 two carbons, 125.4 two carbons, $123.3,114.8$ three carbons, 114.6 three carbons, 59.8, 55.7, 52.1, 45.2, 43.9, 33.7, 31.7, 29.3, 28.2, 22.6, 14.0; IR (neat, $\mathrm{cm}^{-1}$ ) $1720(\mathrm{~m})$; HRMS $\left(\mathrm{ES}^{+}\right)$calcd for $\mathrm{C}_{32} \mathrm{H}_{38} \mathrm{NO}_{3}\left(\mathrm{M}+\mathrm{H}^{+}\right)$, 484.2852, found 484.2855 . 


\section{Coupling to iminoacetates bearing diverse $N$-protecting groups (Reference 11).}

In the Table S-1 below, the results of the screening of three-component coupling reactions with ethyl iminoacetates bearing diverse $\mathrm{N}$-protecting groups are summarized.

Table S-1. Three-component coupling to iminoacetates with variable $N$-protecting groups

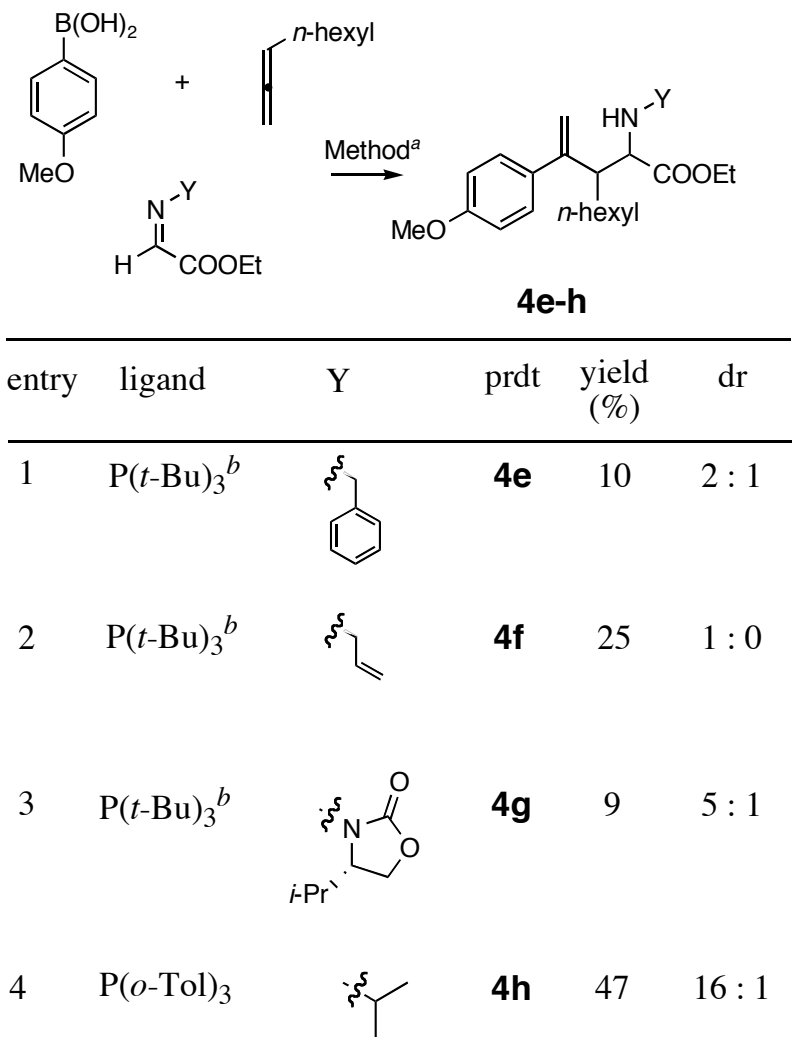

${ }^{a}$ Method: boronic acid : allene $:$ imine = $2: 5: 1$ (mol equiv), $10 \mathrm{~mol} \% \mathrm{Pd}$ cat. $1,10 \mathrm{~mol} \%$ of ligand L, CsF (4.0 equiv), THF, rt, 24 h. The choice of ligand L was optimized by screening with GC/MS monitoring. ${ }^{b}$ The ligand was used as its tetrafluoroborate salt HP( $t$ $\mathrm{Bu})_{3} \mathrm{BF}_{4}$.

Overview of optimization studies in the synthesis of amine 4a (Table 1) (Reference 14).

In the Table S-2 below, the effects of the variations in the reagent ratios, solvent selection, auxiliary phosphine ligand choice and temperature on the yield of amine $4 \mathbf{a}$ are summarized. 
Table S-2. Optimization of the conditions for the preparation of amine $\mathbf{4 a}$

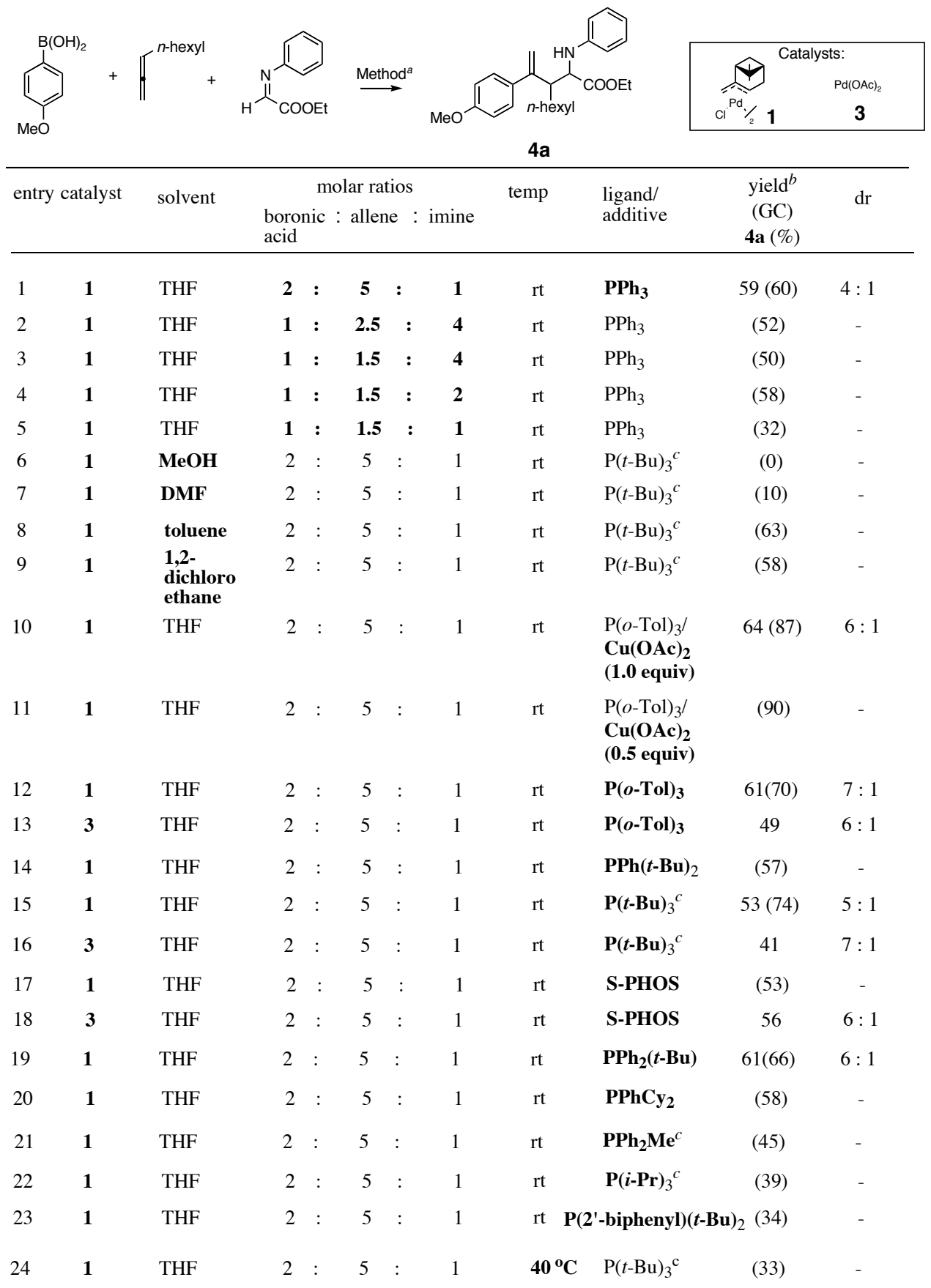

${ }^{a}$ Method: Unless indicated otherwise in the Table, the following conditions were used:10 mol\% Pd cat. 1 or 3, $10 \mathrm{~mol} \%$ of ligand $\mathrm{L}, \mathrm{CsF}$ (4.0 equiv), solvent, $\mathrm{rt}, 24 \mathrm{~h}^{b}{ }^{b}$ The GC/MS yields are given in parentheses, and were determined using undecane as an internal standard. ${ }^{c}$ The ligand was used as its tetrafluoroborate salt $\mathrm{HP}(t-\mathrm{Bu})_{3} \mathrm{BF}_{4}$. 
Monitoring the three-component coupling reactions by TLC and GC/MS indicated that the initial rate of formation of the homoallylic amines was greater in the presence of electron deficient phosphine ligands (i.e. $\mathrm{PPh}_{3}$ and $\left.\mathrm{P}(o-\mathrm{Tol})_{3}\right)$ as compared to electron rich phosphine ligands (i.e. $\mathrm{P}(t-\mathrm{Bu})_{3}, \mathrm{PCy} y_{3}$, and $\left.\mathrm{PPh}(t-\mathrm{Bu})_{2}\right)$. However, reactions typically reached similar conversions over a period of $24 \mathrm{~h}$ as indicated by both GC/Ms of crude reactions mixtures and isolated yields of the homoallylic amines.

\section{Formation of amine 4a under Pd(0) catalysis (Reference 15).}

Treatment of $p$-methoxyphenylboronic acid ( $0.068 \mathrm{~g}, 0.450 \mathrm{mmol}, 2.0$ equiv), CsF (0.137 g, $0.900 \mathrm{mmol}, 4.0$ equiv), ethyl $N$-phenyliminoacetate ( $0.040 \mathrm{~g}, 0.225 \mathrm{mmol}, 1.0$ equiv) and 1,2-nonadiene $\left(0.140 \mathrm{~g}, 1.13 \mathrm{mmol}, 5.0\right.$ equiv) with $\mathrm{Pd}_{2} \mathrm{dba}_{3}(0.010 \mathrm{~g}, 0.011 \mathrm{mmol}$, 0.1 equiv $\mathrm{Pd})$ and $\mathrm{H}\left[\mathrm{P}(t-\mathrm{Bu})_{3}\right] \mathrm{BF}_{4}(0.007 \mathrm{~g}, 0.023 \mathrm{mmol}, 0.1$ equiv) at $\mathrm{rt}$ according to the general procedure described above followed by flash chromatography over silica eluting with EtOAc/hexane $(1: 20)$ afforded amine $4 \mathbf{a}(0.020 \mathrm{~g}, 22 \%)$ as a mixture of inseparable diastereomers ( $\mathrm{dr} 7: 1)$ as a colorless oil.

\section{NOE ${ }^{1} \mathrm{H}$ NMR studies on tetrahydropyridines $14 \mathrm{a}$ and $14 \mathrm{~b}$.}

In an attempt to elucidate the relative stereochemistry in tetrahydropyridines $\mathbf{1 4}$, selective NOE spectra were recorded, and the results are summarized below.

Compound 14a: Irradiation of the proton $\mathrm{H}^{2}(4.59 \mathrm{ppm}(\mathrm{d}, J=2.0 \mathrm{~Hz}, 1 \mathrm{H}))$ resulted in the enhancement of the signals for the proton $\mathrm{H}^{3}(3.27 \mathrm{ppm}(\mathrm{d} \mathrm{br}, J=7.6 \mathrm{~Hz}, 1 \mathrm{H}))$. Also, irradiation of the proton $\mathrm{H}^{3}$ resulted in the enhancement of the signals for the proton $\mathrm{H}^{2}$. 


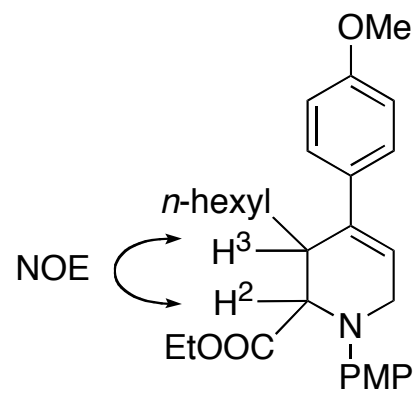

$14 a$

$\mathrm{H}^{2}: 4.59(\mathrm{~d}, \mathrm{~J}=2.0 \mathrm{~Hz}, 1 \mathrm{H})$

$\mathrm{H}^{3}: 3.27(\mathrm{~d} \mathrm{br}, J=7.6 \mathrm{~Hz}, 1 \mathrm{H})$

Compound 14b: Irradiation of the proton $\mathrm{H}^{2}(5.07 \mathrm{ppm}(\mathrm{s}$ br, $1 \mathrm{H})$ ) resulted in the enhancement of the signals for the proton $\mathrm{H}^{3}(3.14 \mathrm{ppm}(\mathrm{d} \mathrm{br}, J=10.2 \mathrm{~Hz}, 1 \mathrm{H}))$. Also, irradiation of the proton $\mathrm{H}^{3}$ resulted in the enhancement of the signals for the proton $\mathrm{H}^{2}$.

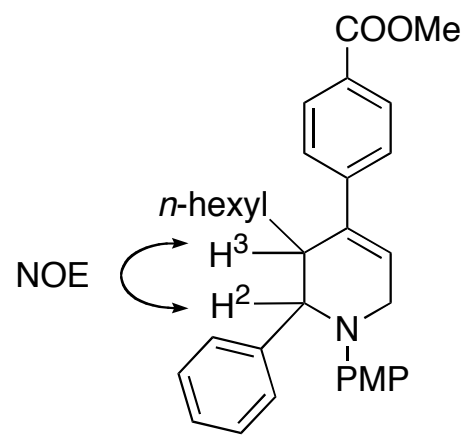

$14 b$

$\mathrm{H}^{2}: 5.07(\mathrm{~s} \mathrm{br}, 1 \mathrm{H})$

$\mathrm{H}^{3}: 3.14(\mathrm{~d} \mathrm{br}, J=10.2 \mathrm{~Hz}, 1 \mathrm{H})$

However, computational analysis (molecular dynamics simulation) performed on trans isomers of tetrahydropyridines indicated that the distance of protons $\mathrm{H} 2$ and $\mathrm{H} 3$ oscillates around $3 \AA$, and consequently it can be expected that both trans and cis diastereomers would generate a detectable NOE signal between $\mathrm{H} 2$ and $\mathrm{H} 3$. 


\section{References.}

1 Moreaou, J.-L.; Gaudemar, M. J. Organomet. Chem. 1976, 108, 159-164.

2 Hosokawa, T.; Uno, T.; Inui, S.; Murahashi, S.-I. J. Am. Chem. Soc. 1981, 103, $2318-2323$.

3 Manhas, M. S.; Ghosh, M.; Bose, A. K. J. Org. Chem. 1990, 55, 575-580.

4 a) Chacun-Lefevre, L.; Beneteau, V.; Joseph, B.; Merour, J.-Y. Tetrahedron 2002, 58, 10181-10188. b) Barluenga, J.; Fañanás, F. J.; Sanz, R.; Fernández, Y. Chem. Eur. J. 2002, 8, 2034-2046.

5 Donohoe, T. J.; Orr, A. J.; Gosby, K.; Bingham, M. Eur. J. Org. Chem. 2005, 1969-1971. 


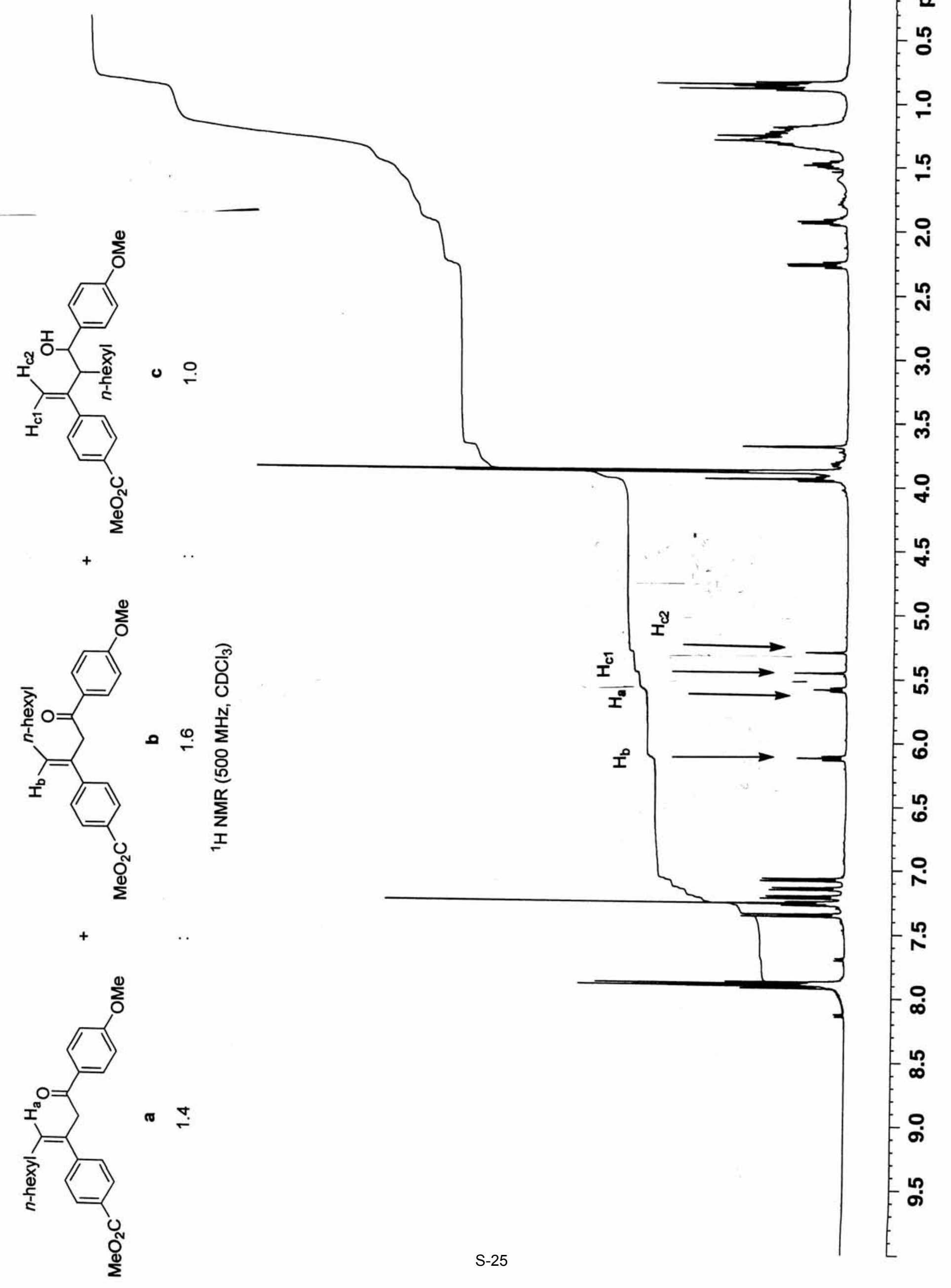




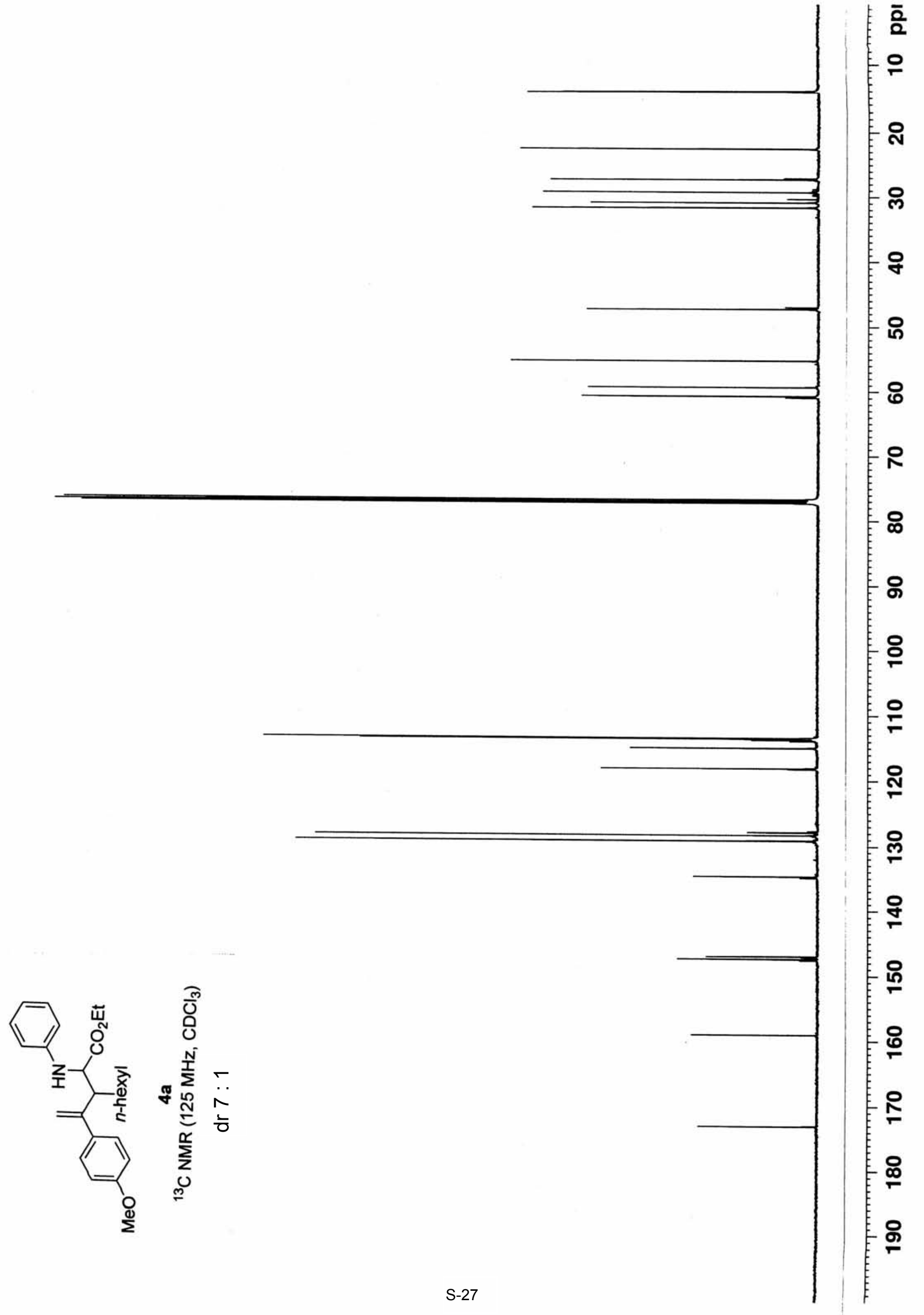




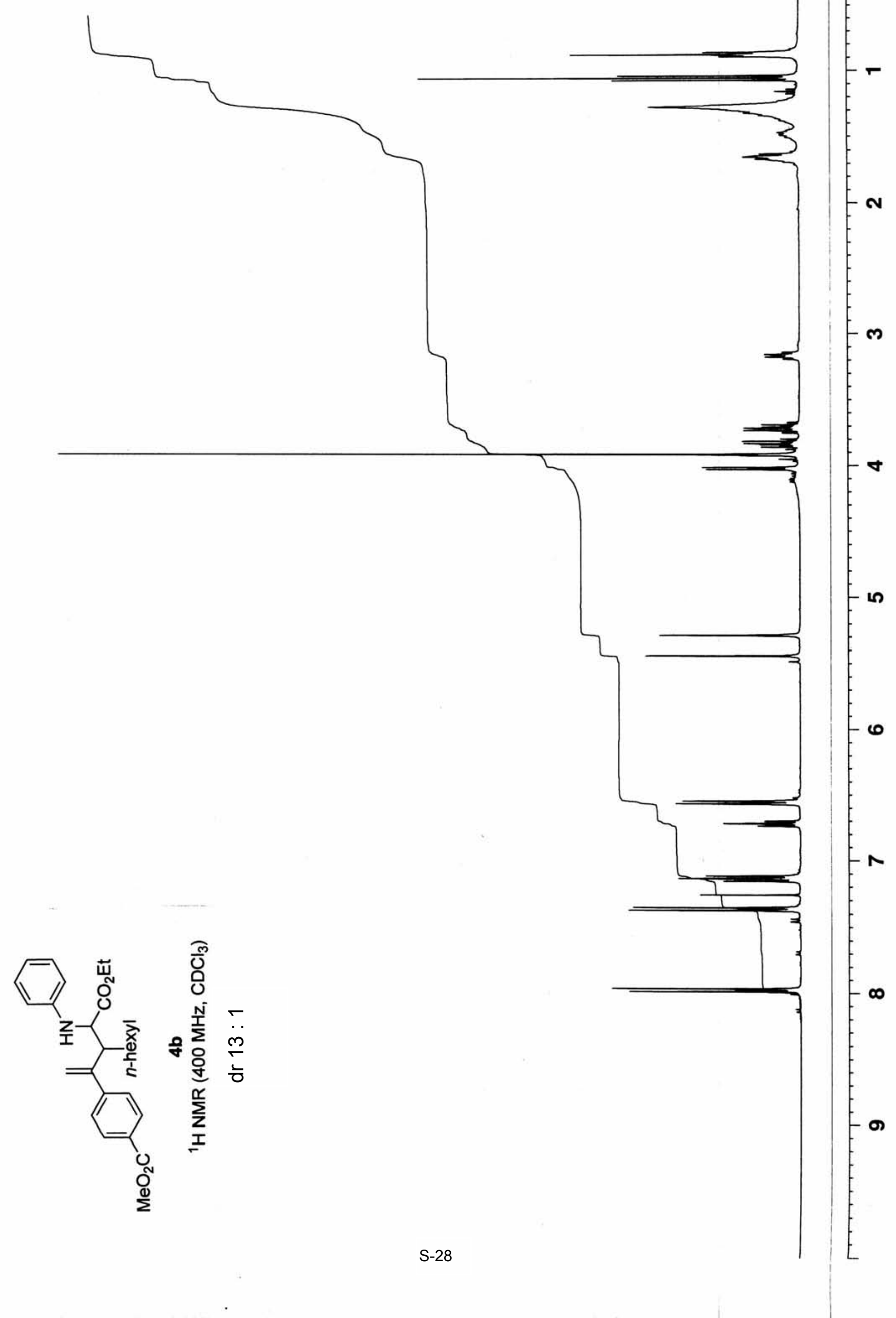




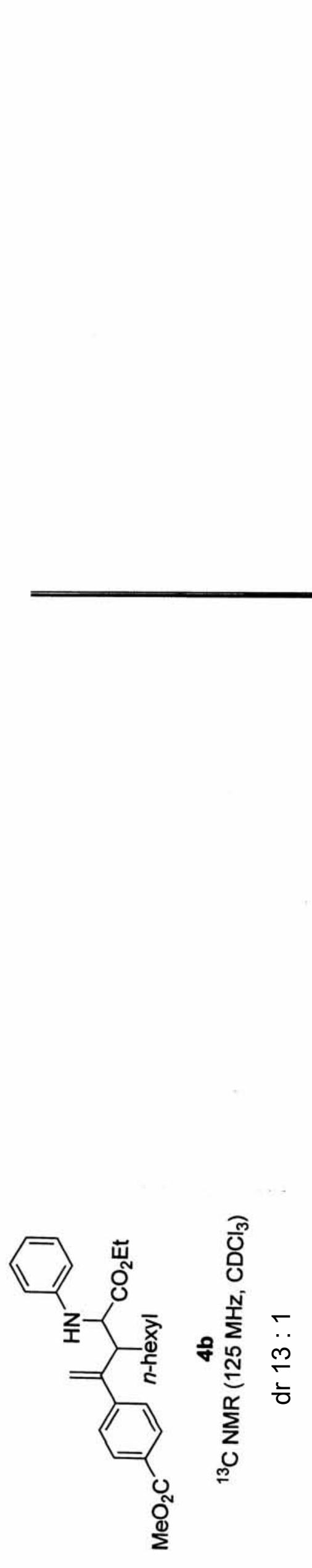

$\frac{2}{2}$ 


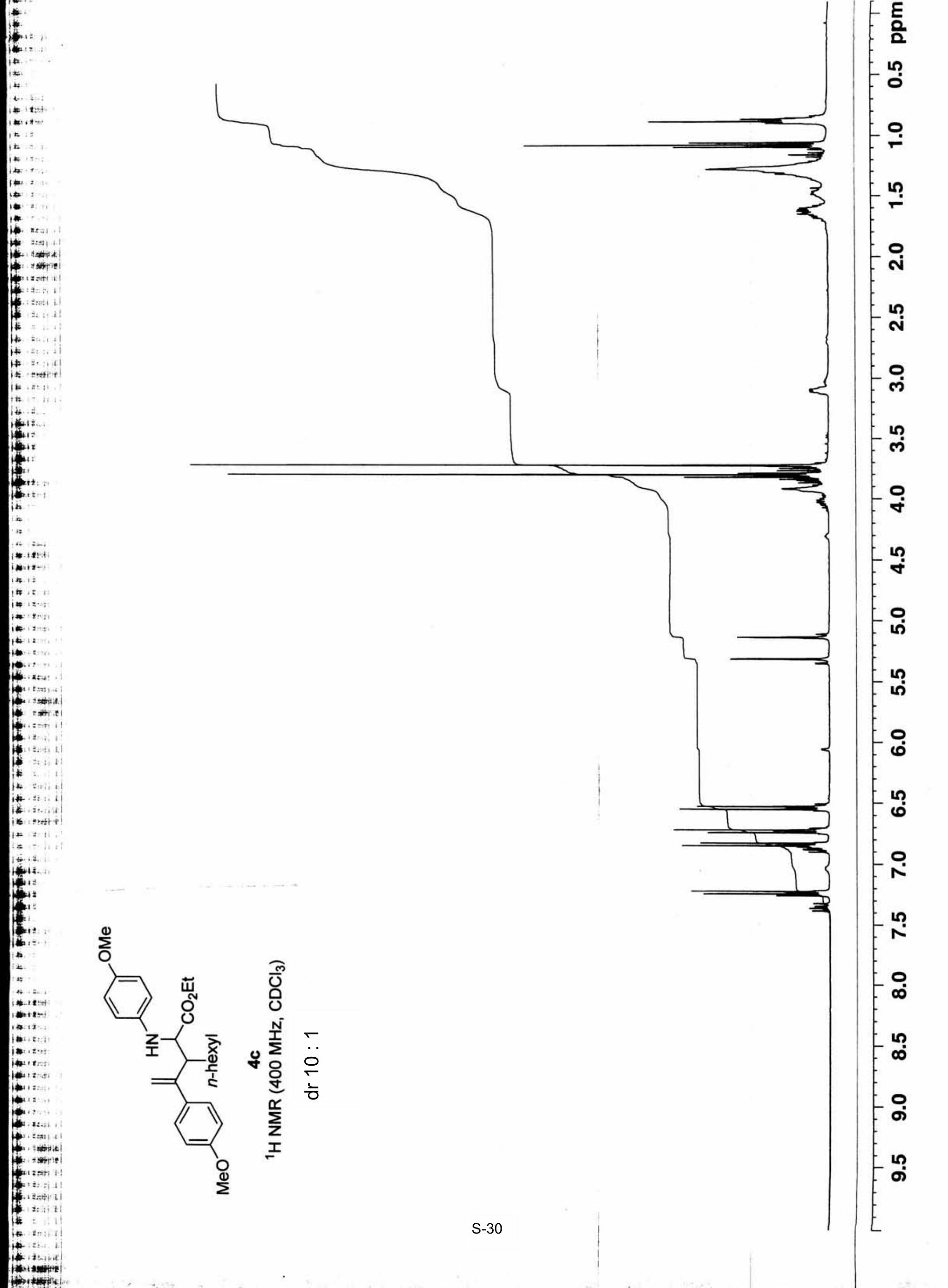


in:

Qin:

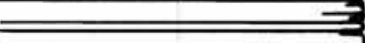

政

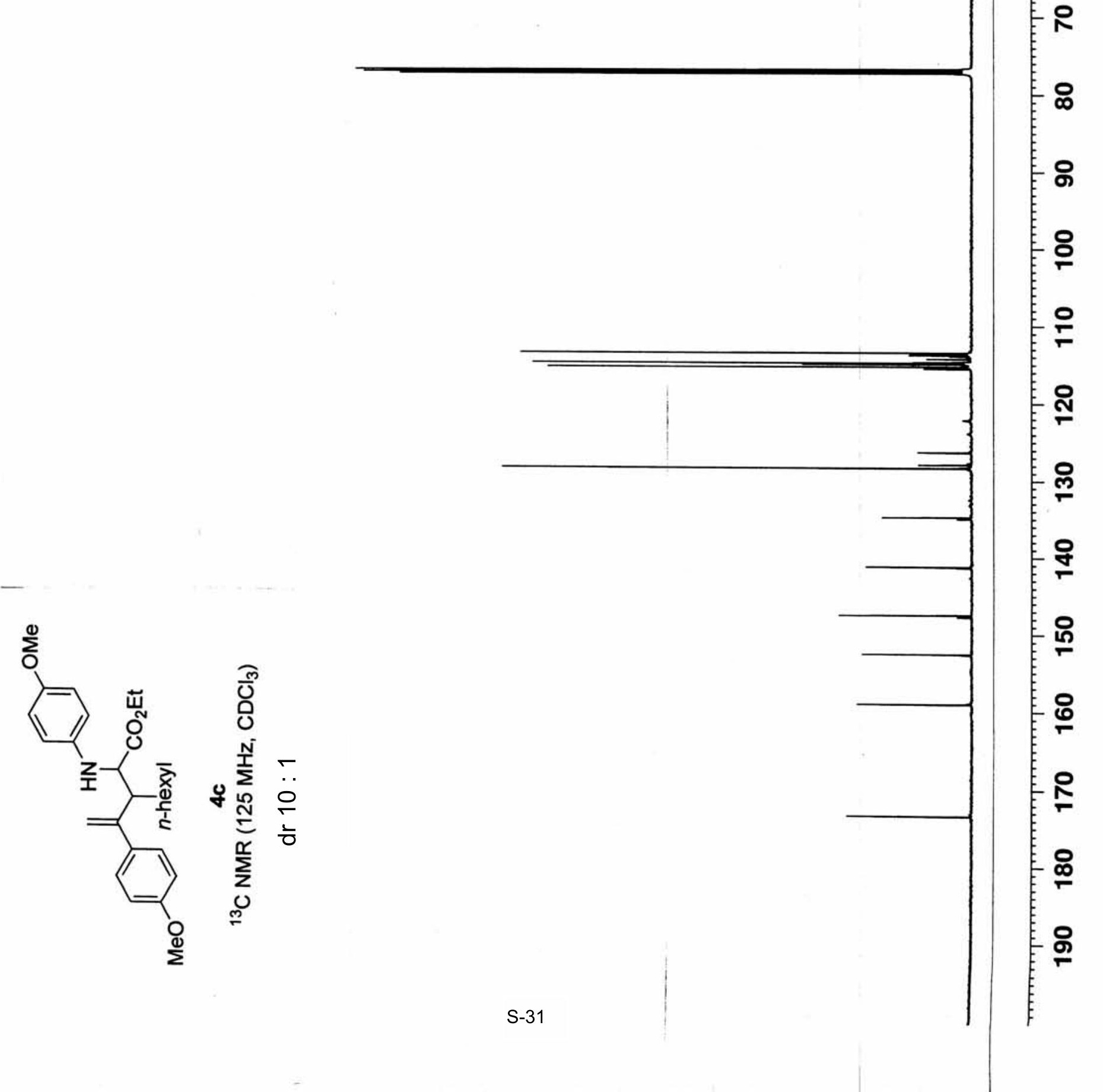




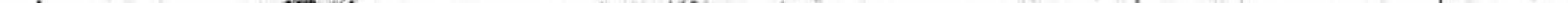




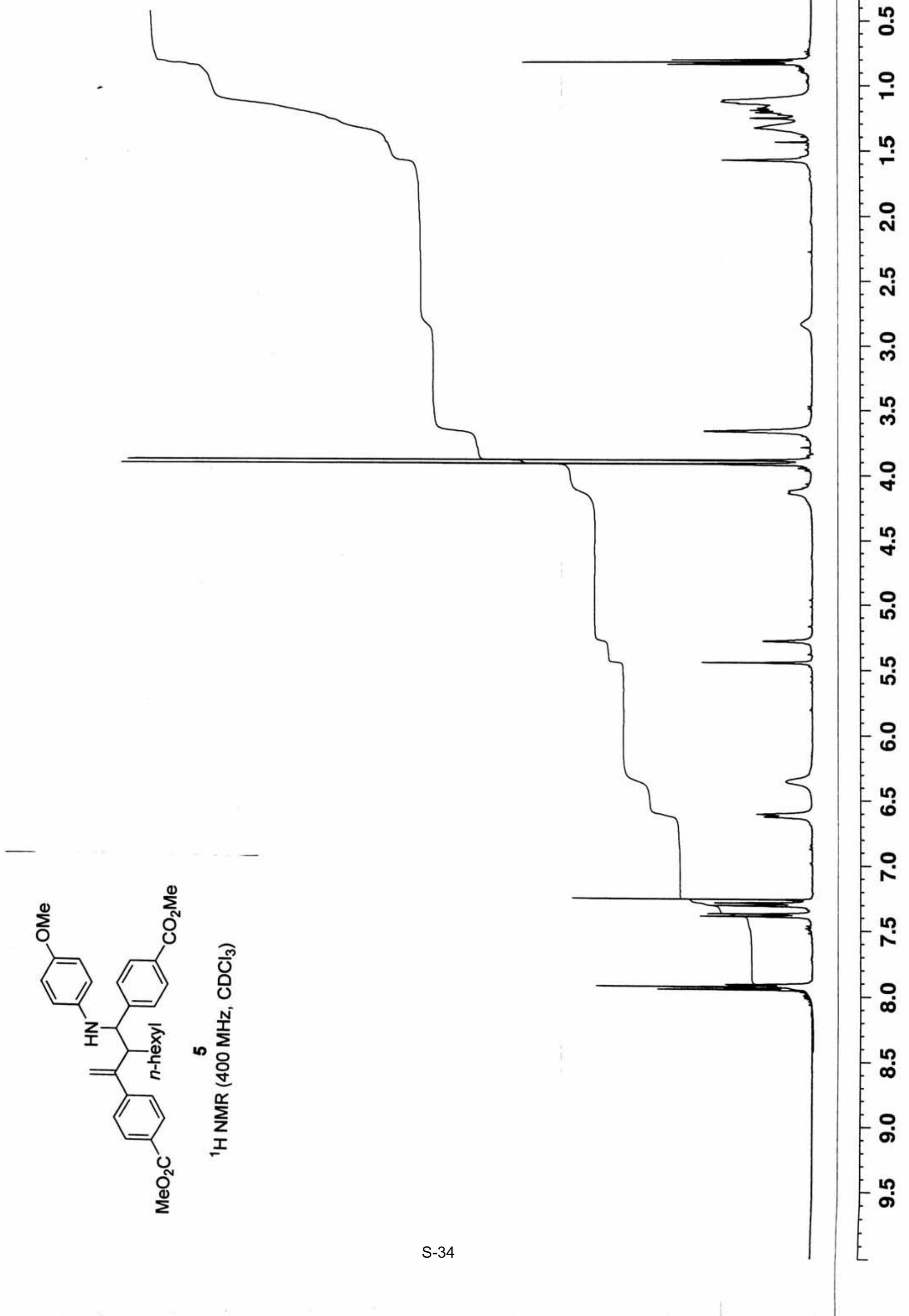




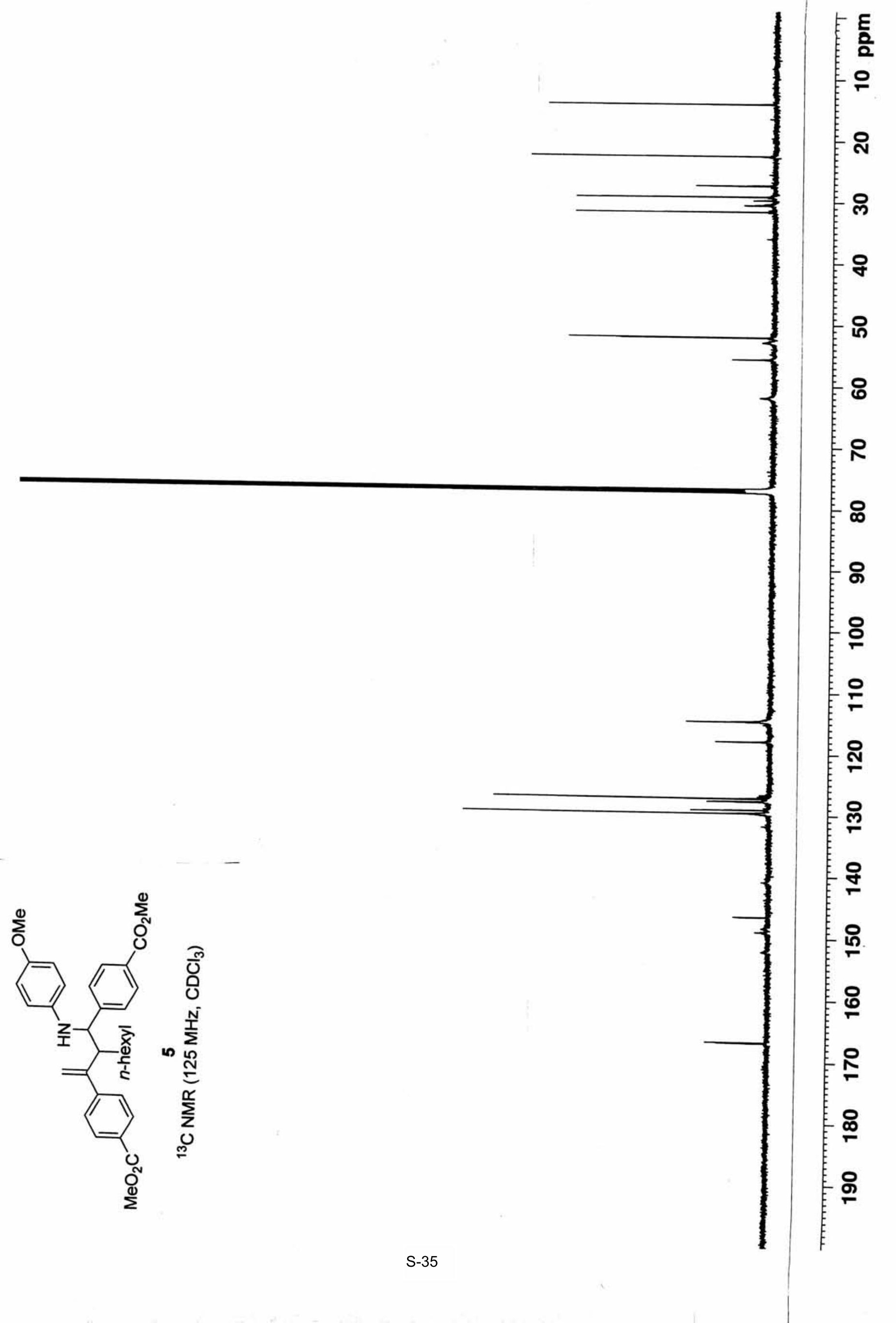




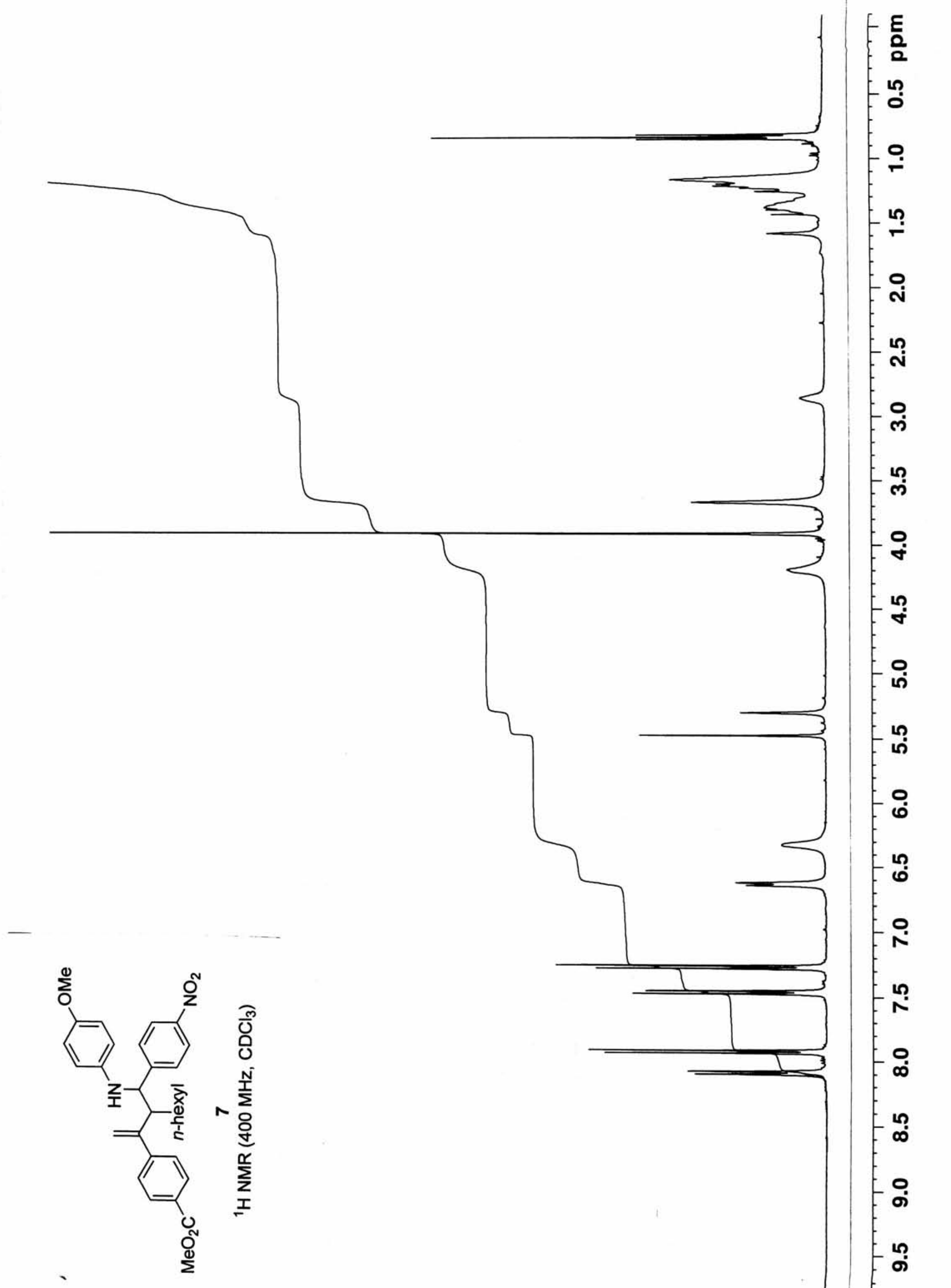

$f \frac{E}{2}$

?

- 은

- 우

웅

ก

ल

ल

웅

?

-

- 18

-

-

-

-

N

$-\infty$ 


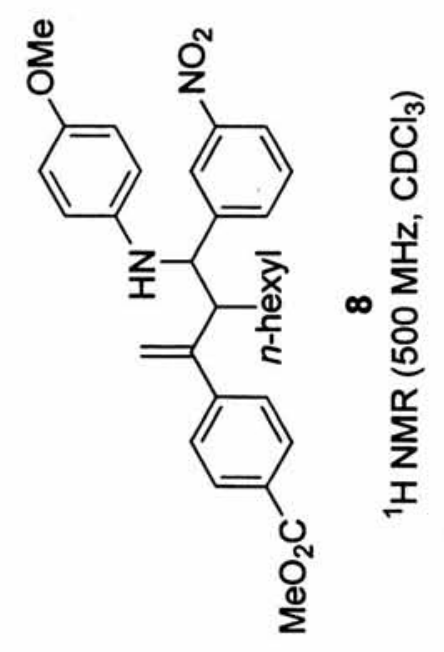


을

- 으

$=$ 오

-

- 웅

$=$ 우

$-8$

요

$=\infty$

-

응

$=$

$=$ 온

i

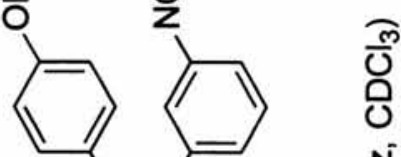

=1

గ్ల 


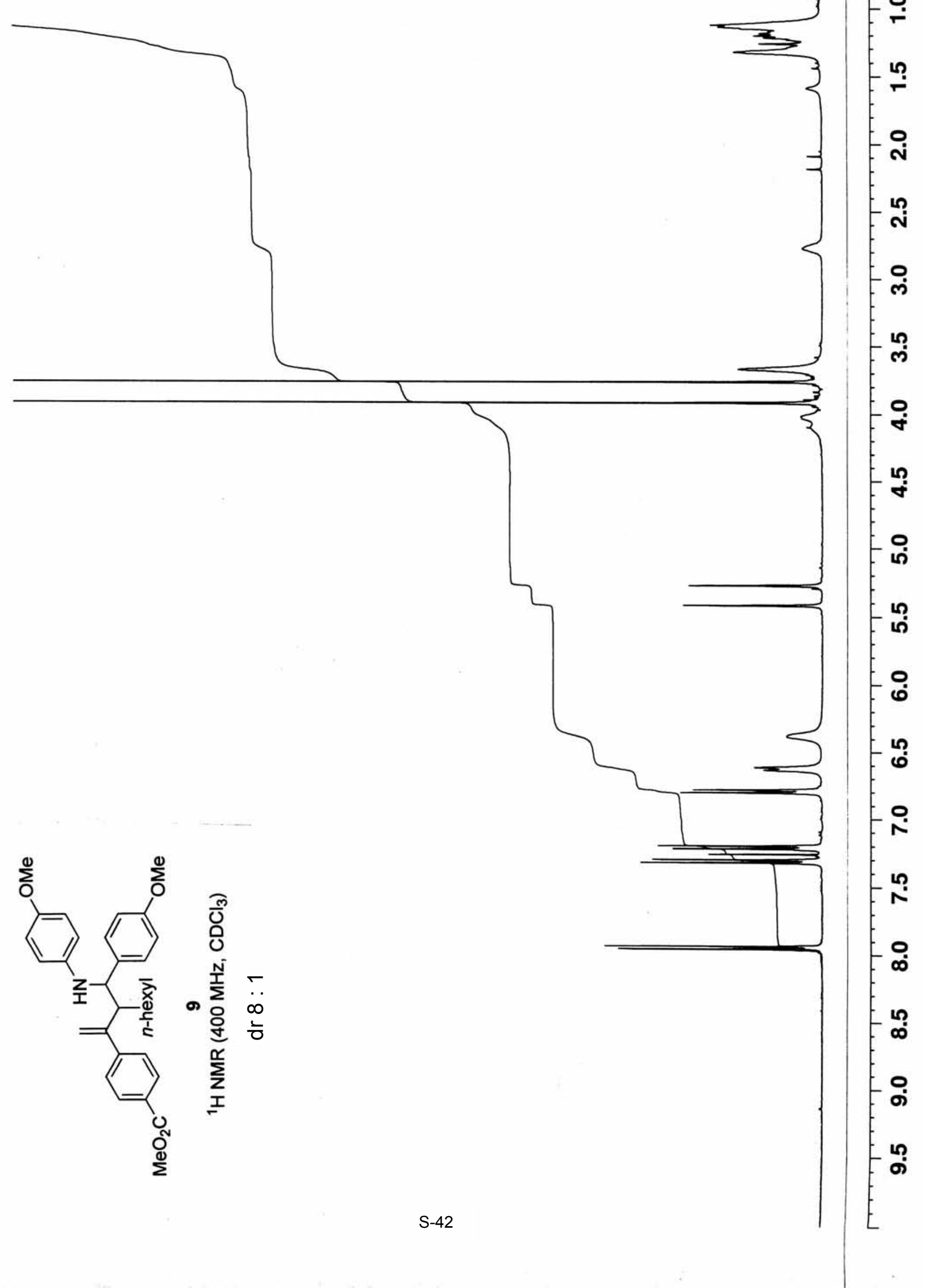




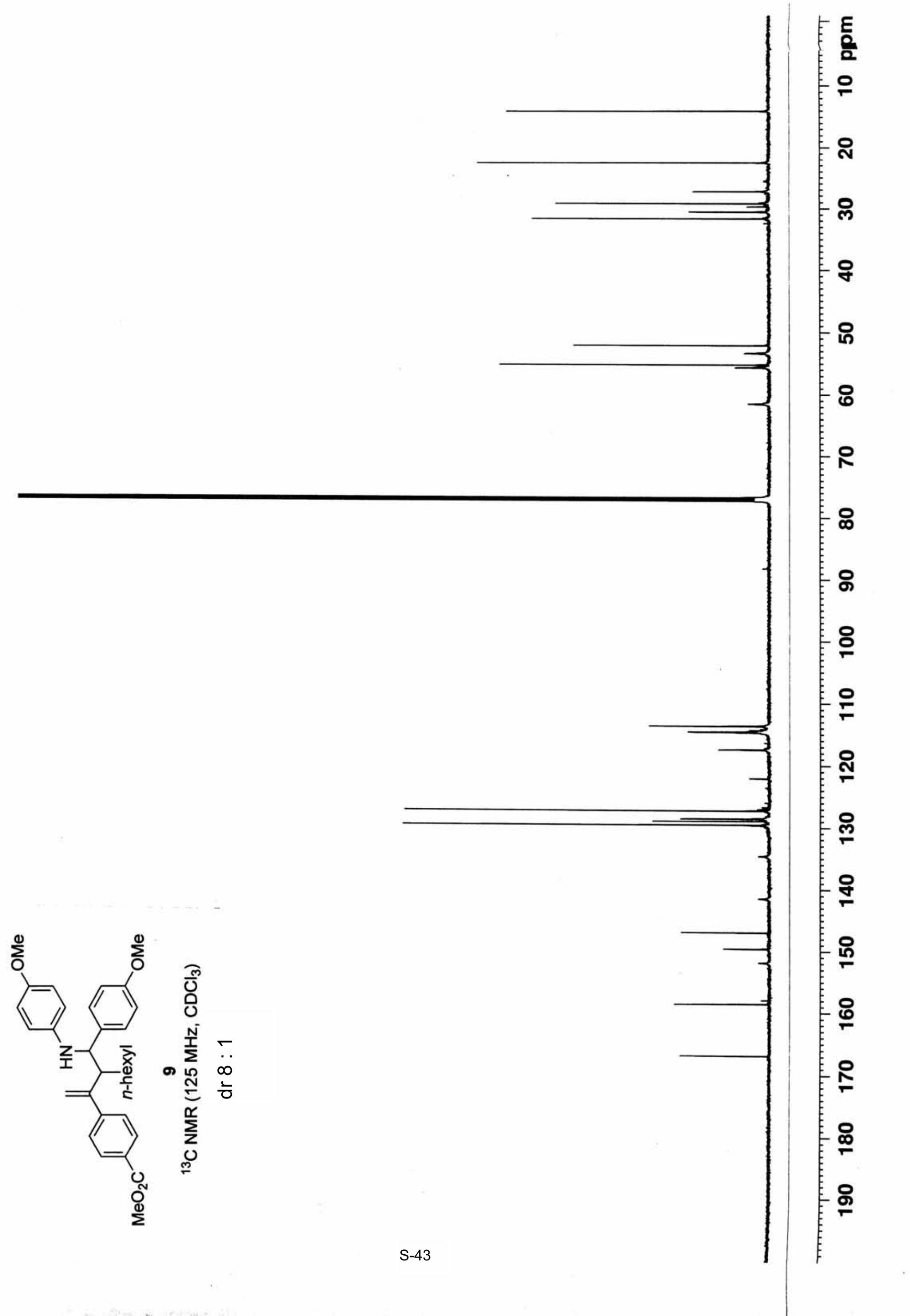




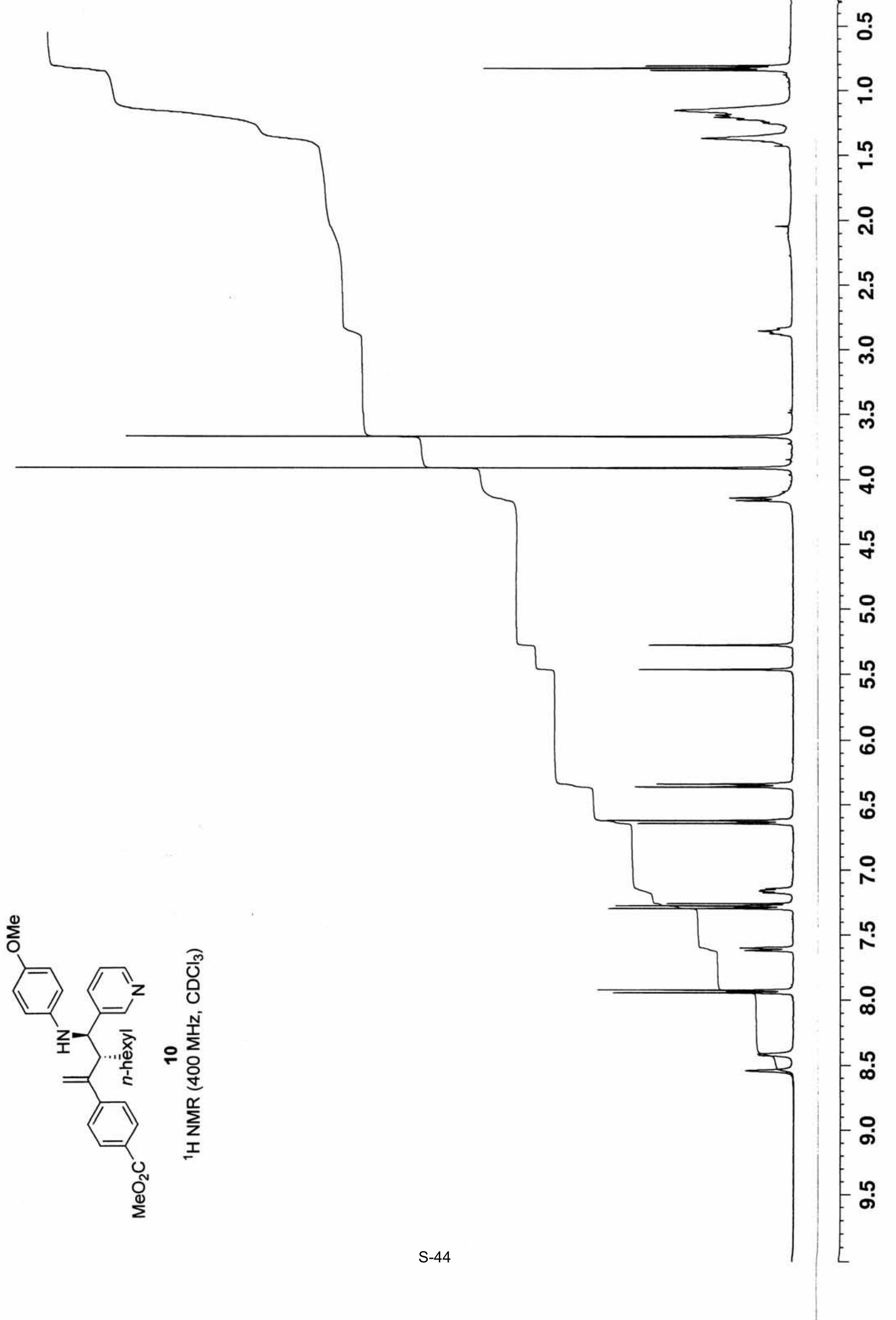



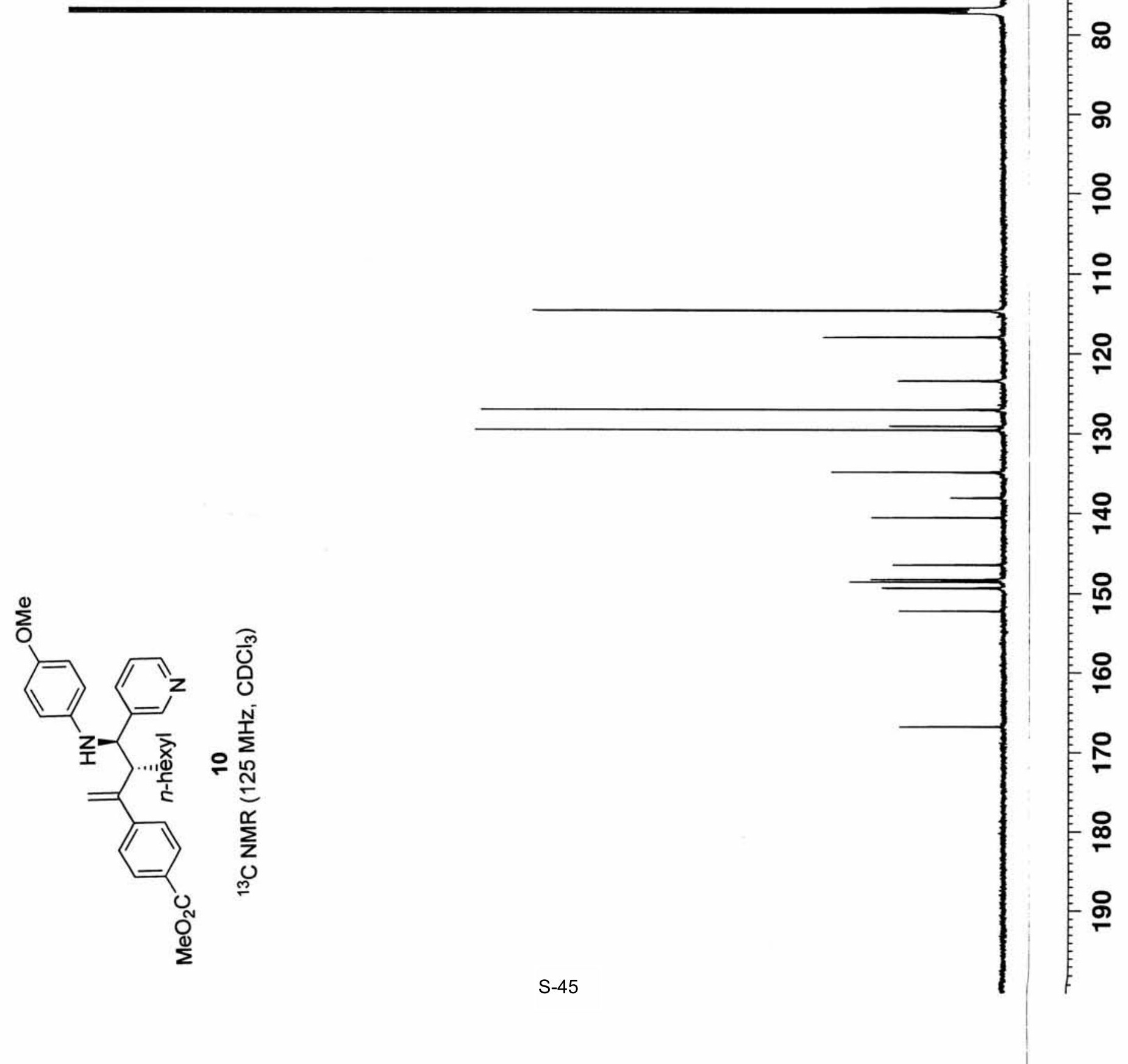


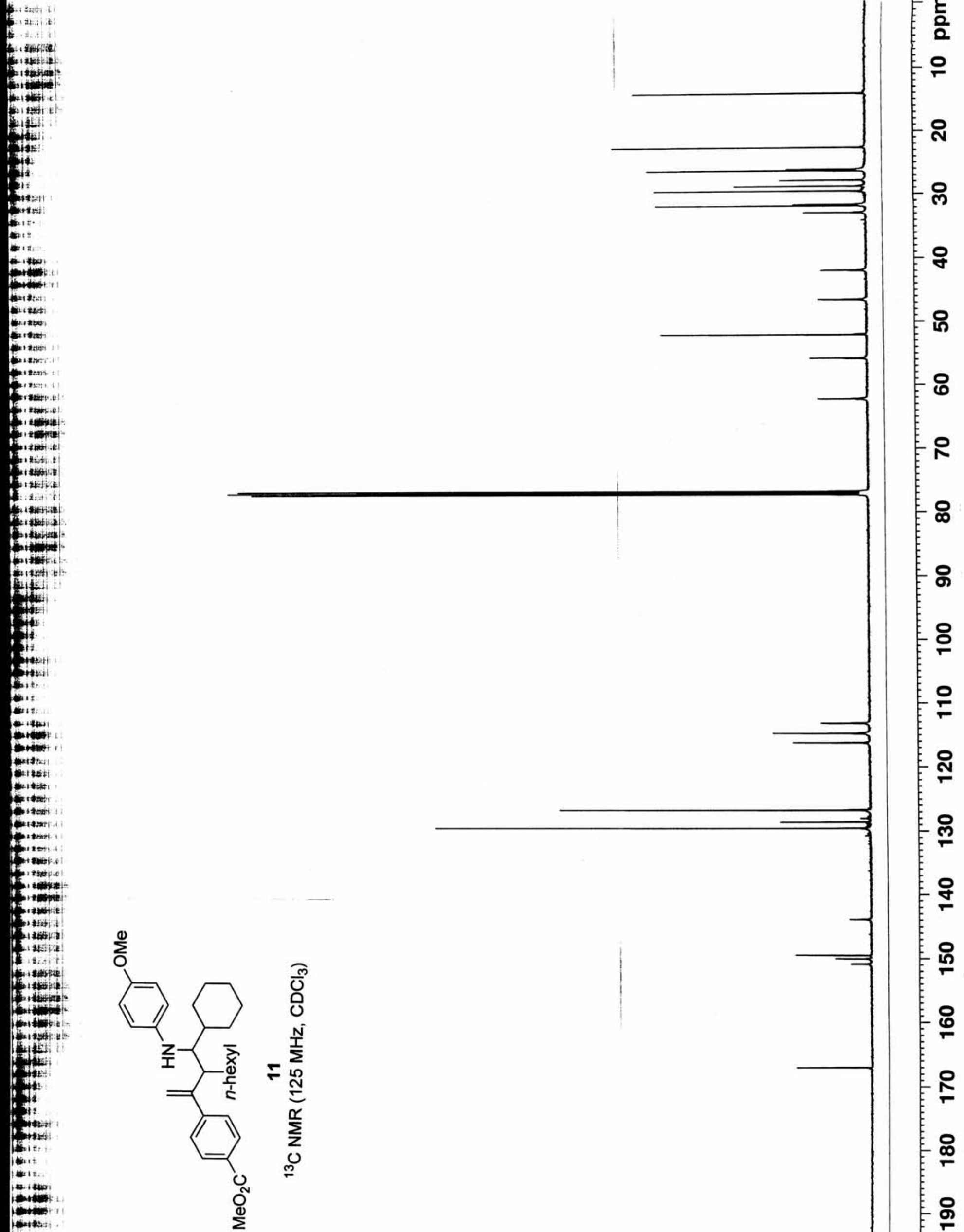




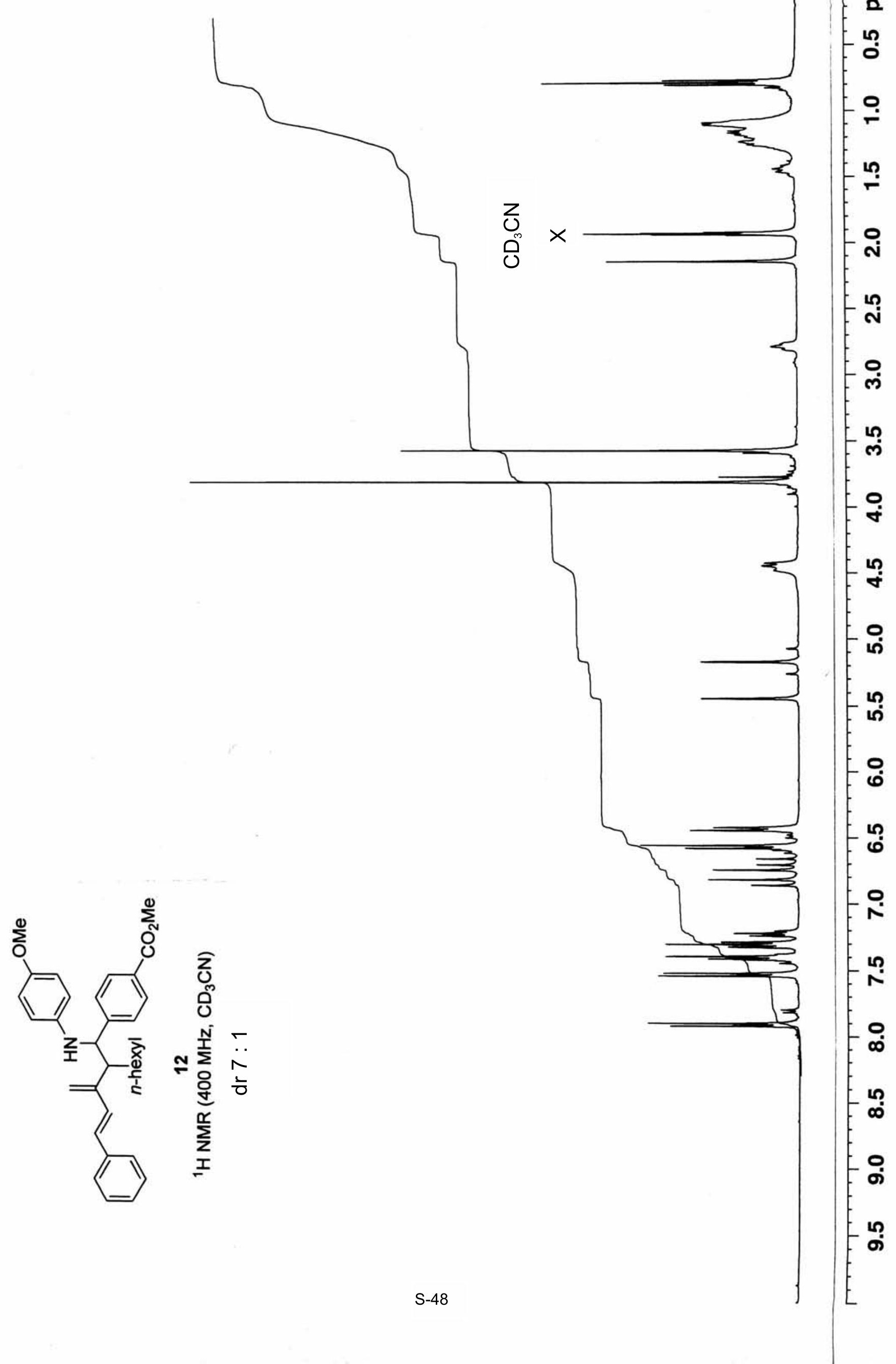




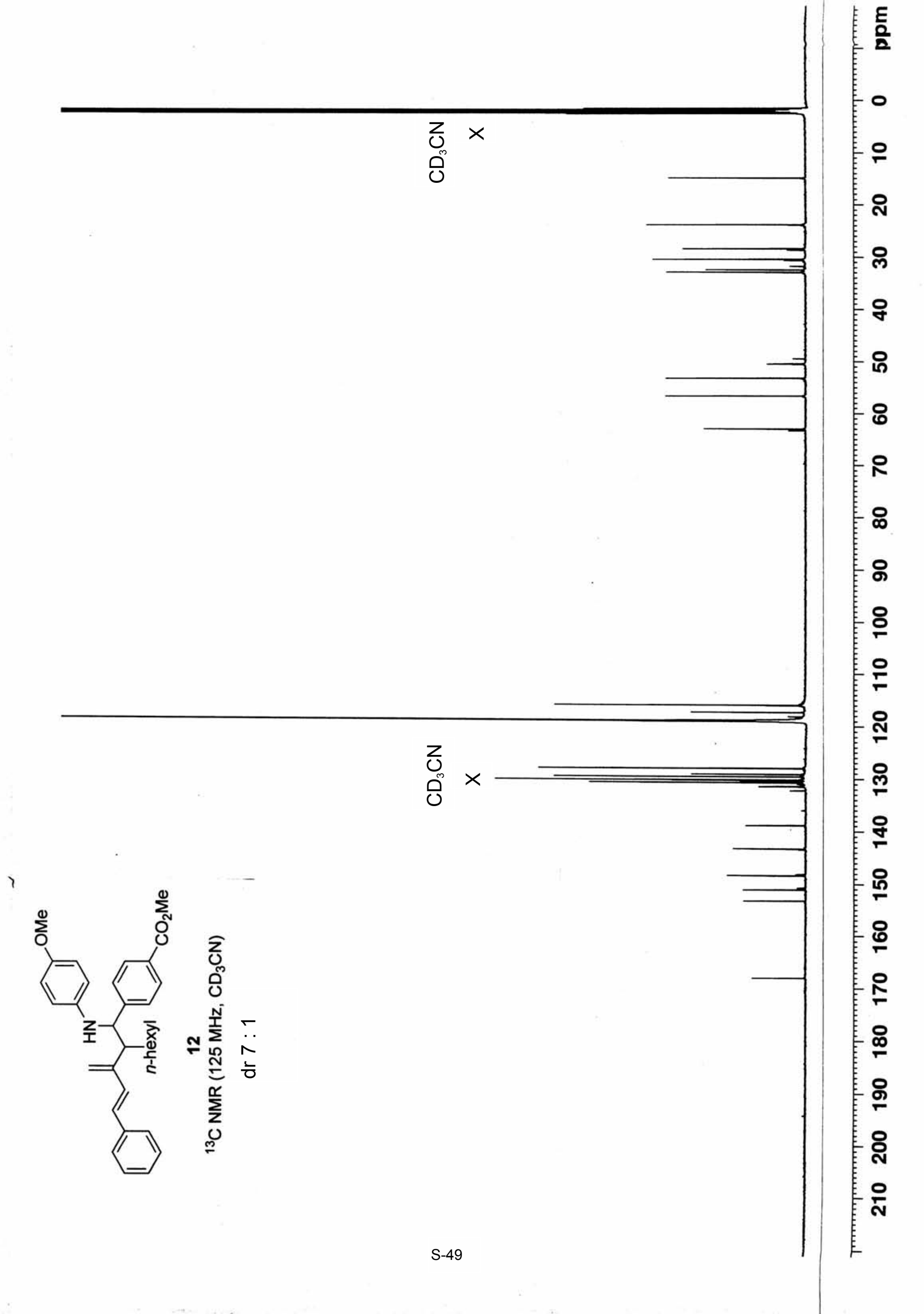




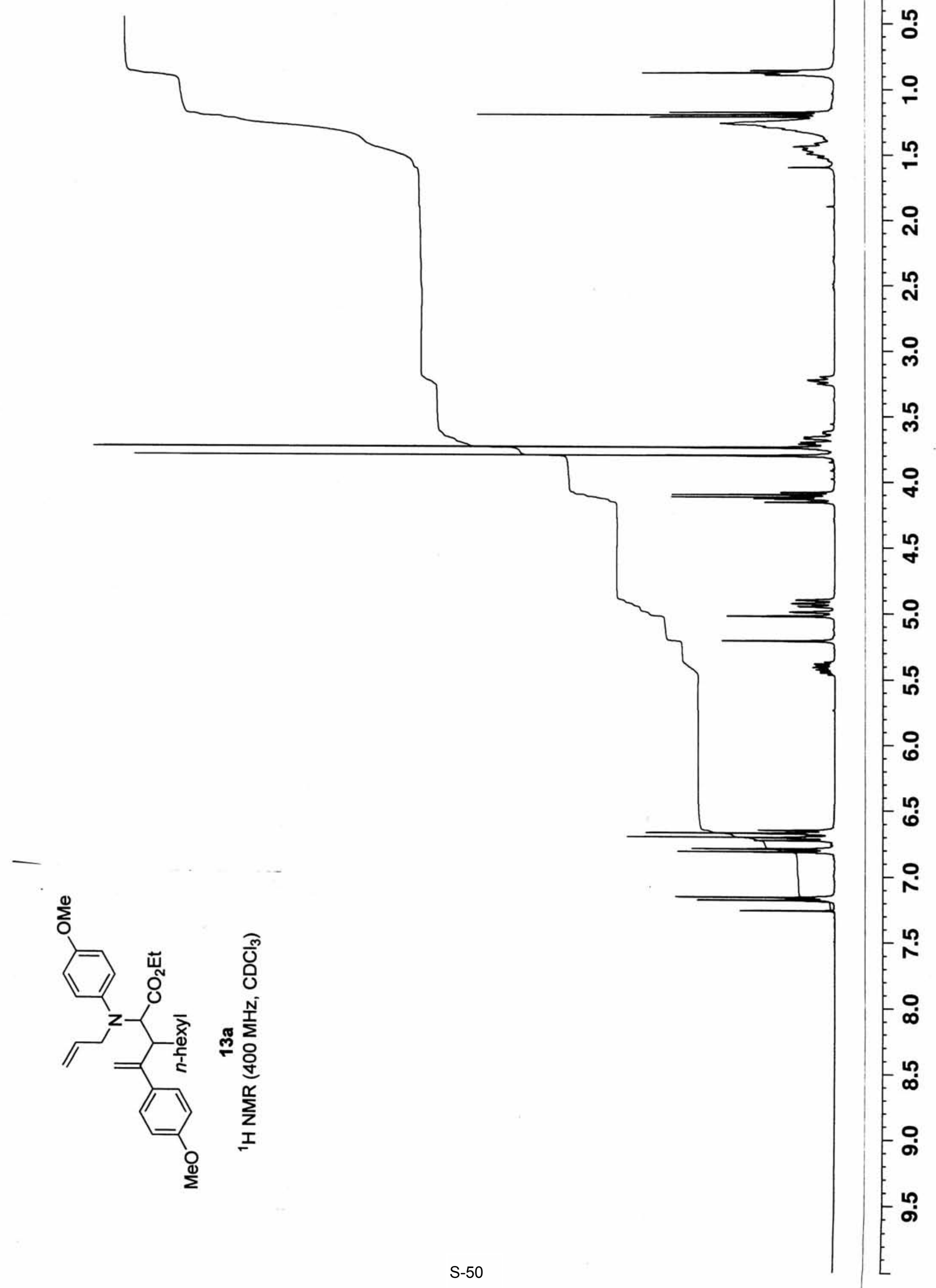




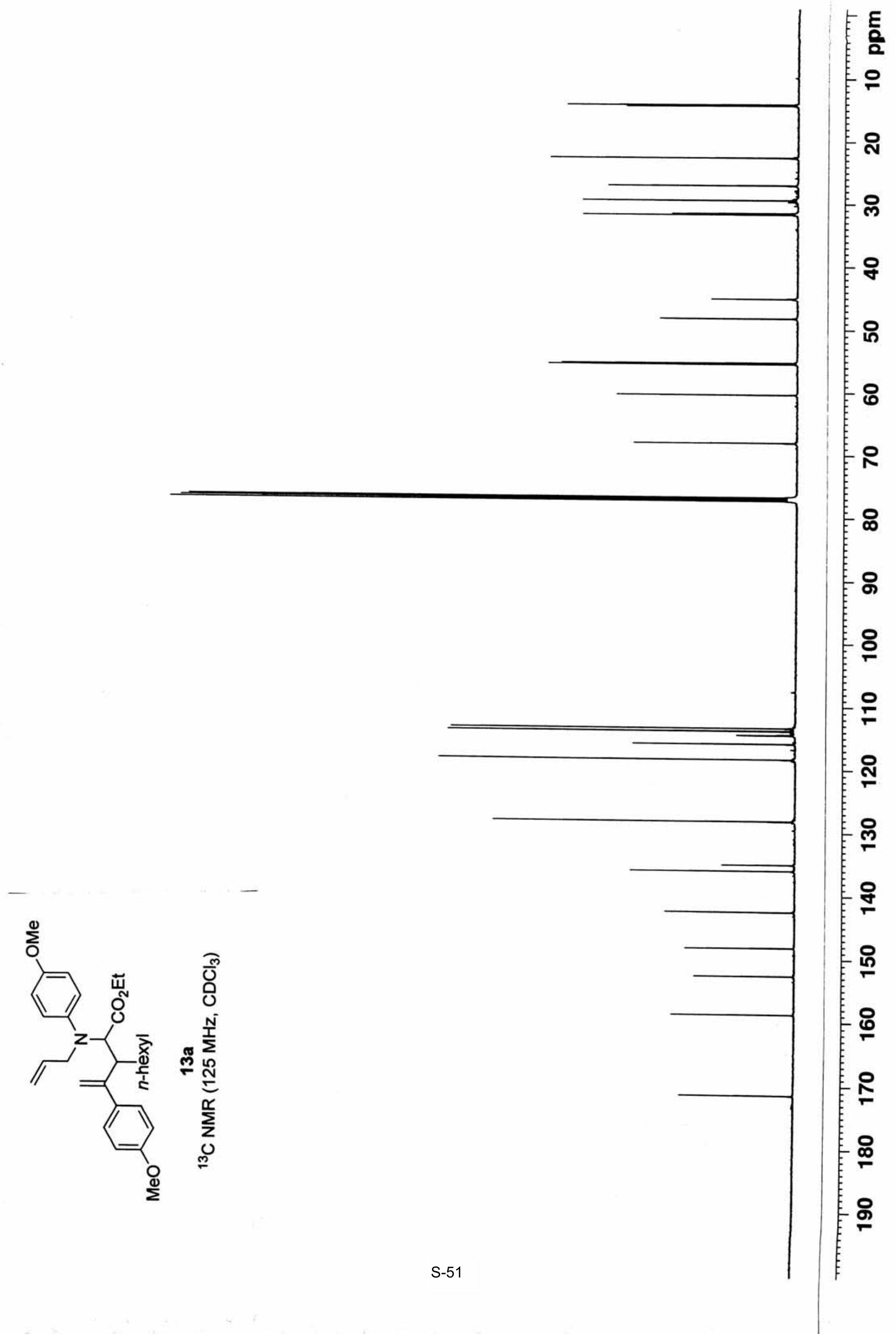




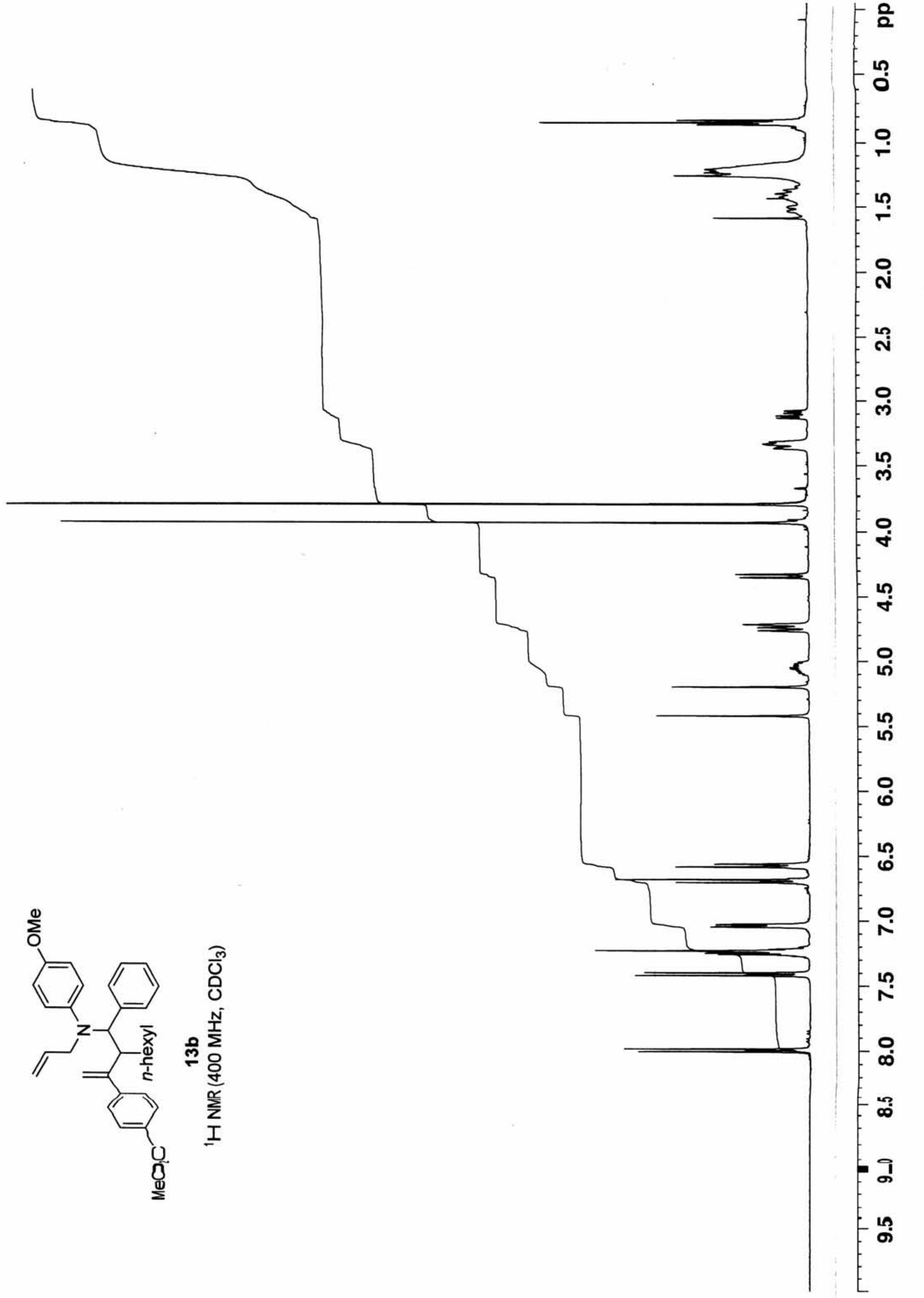




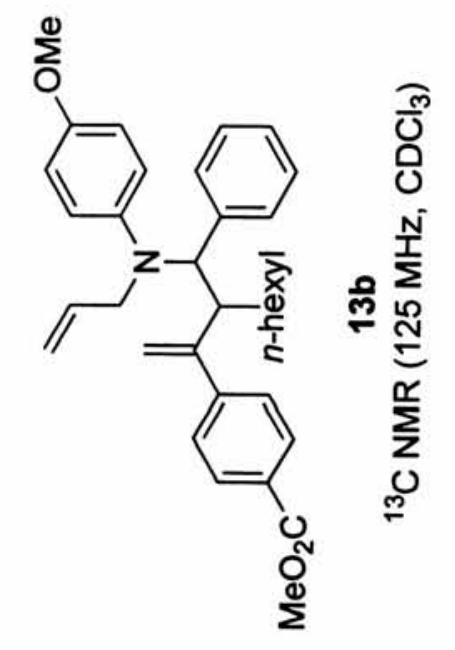




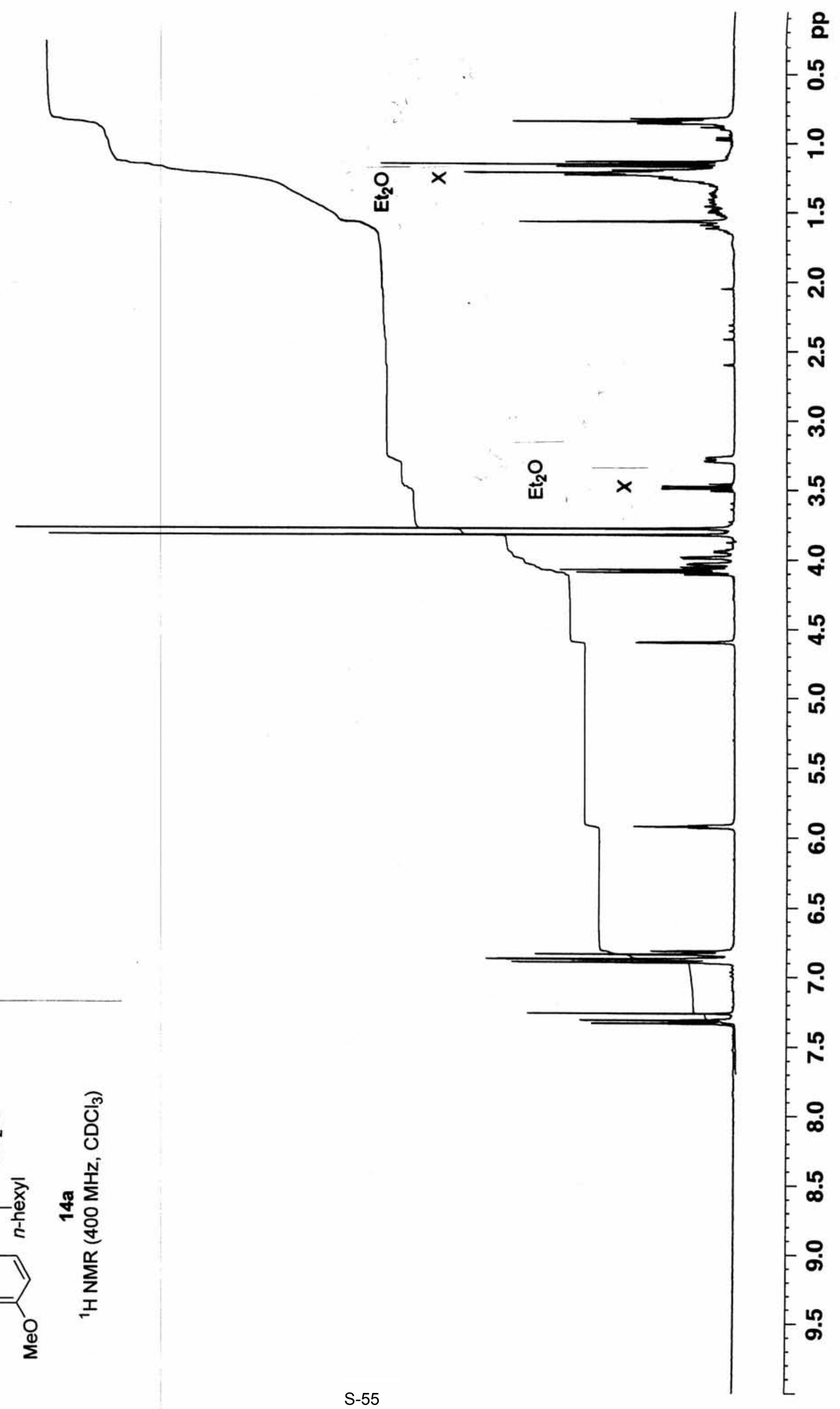




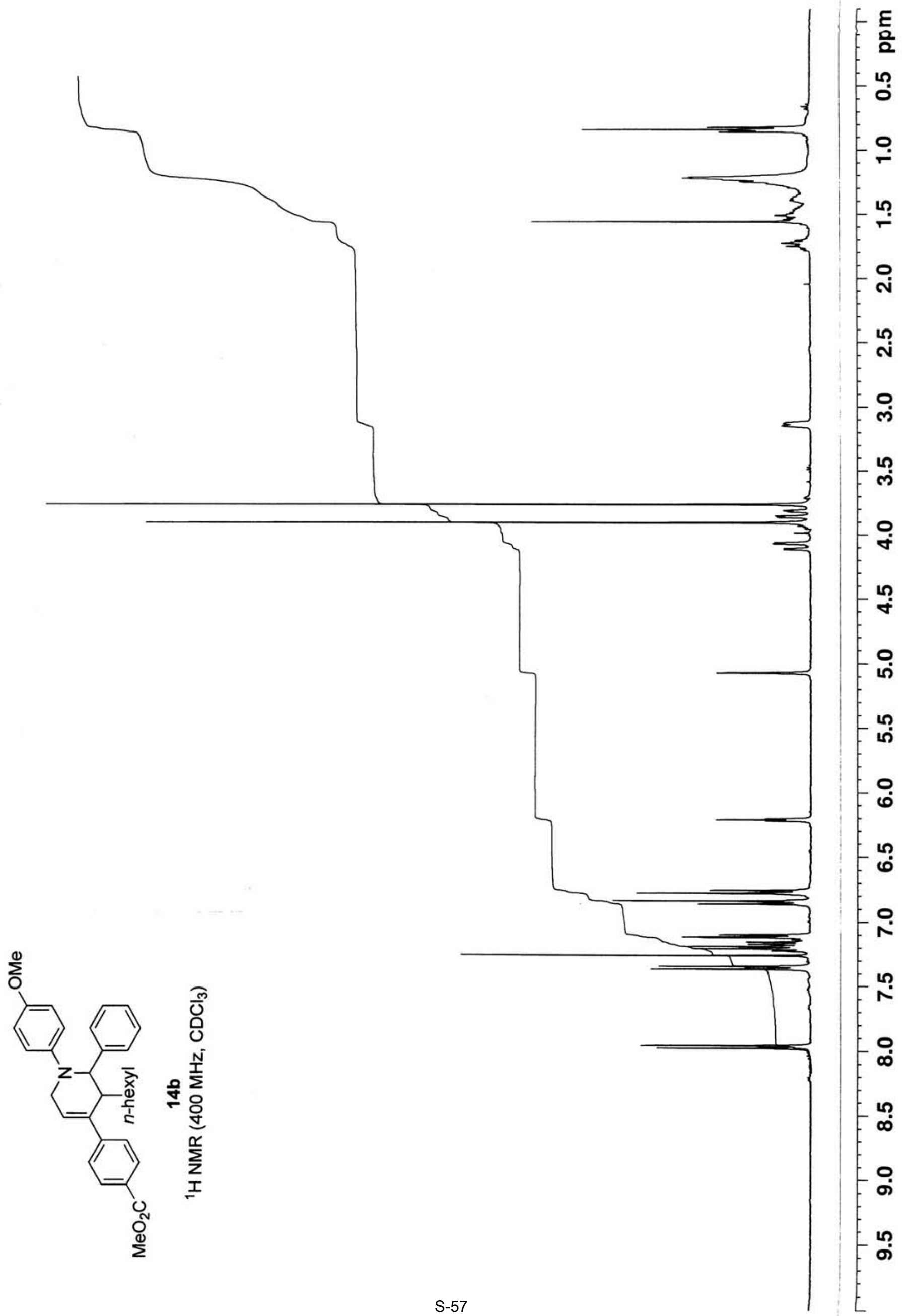



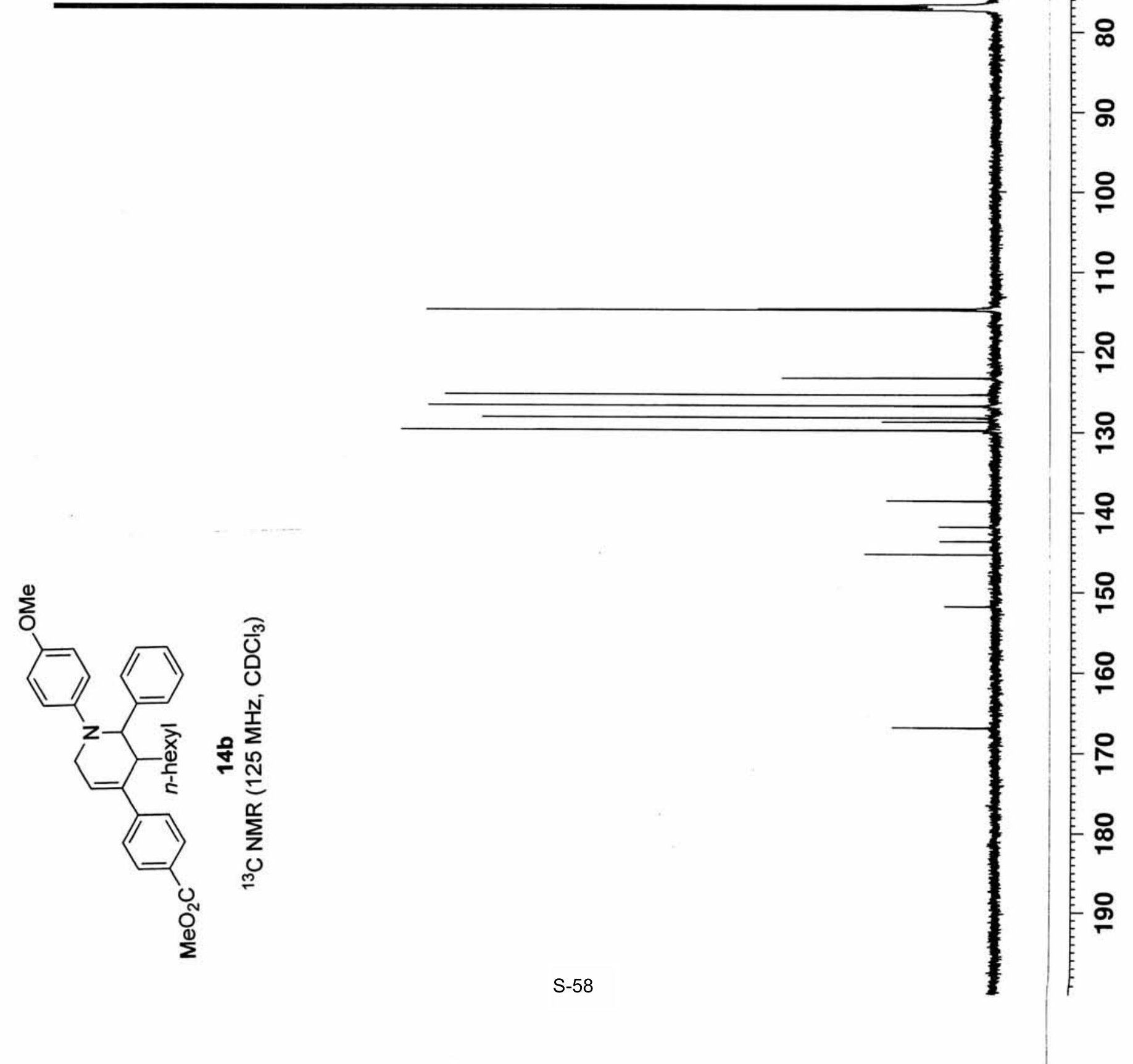


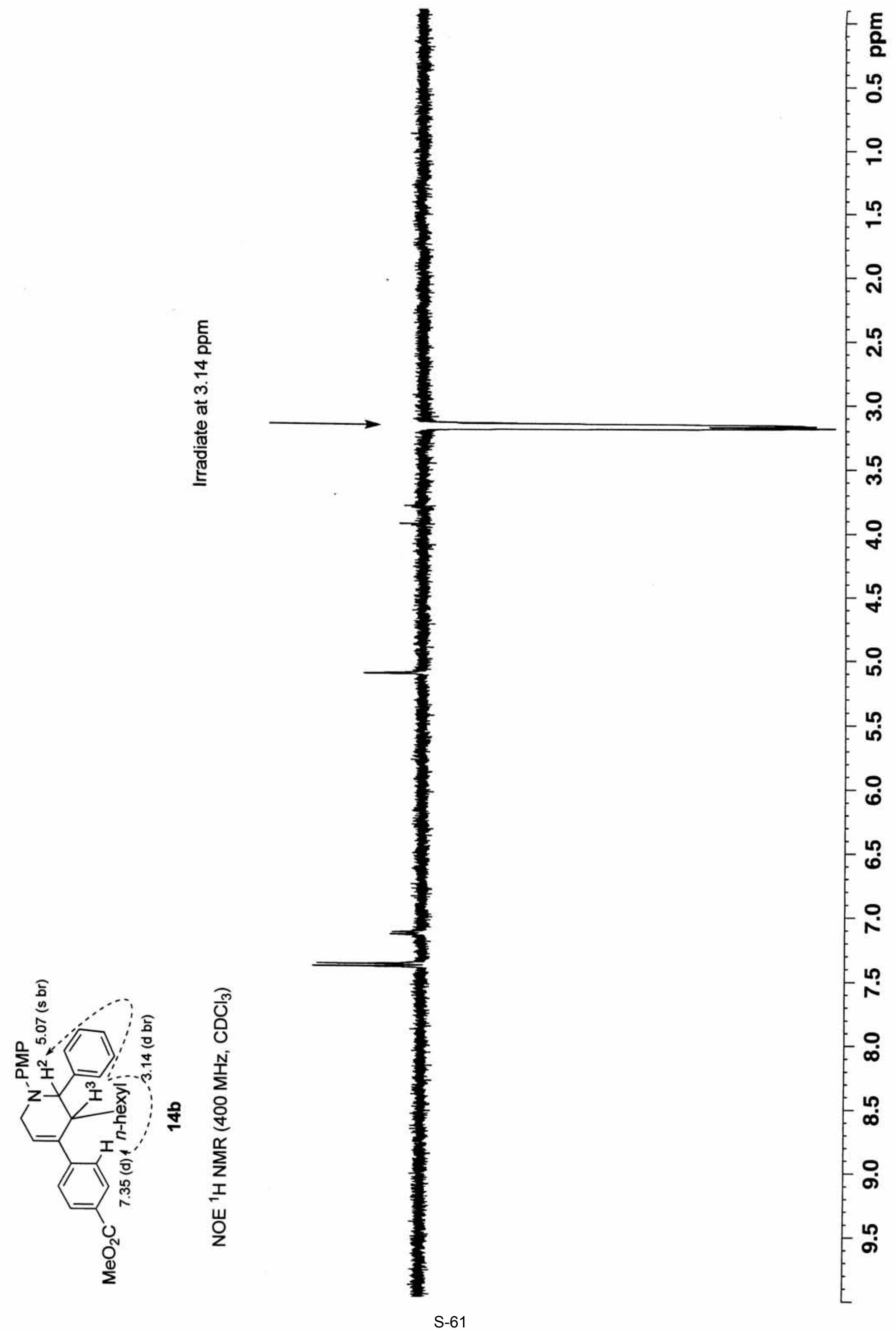




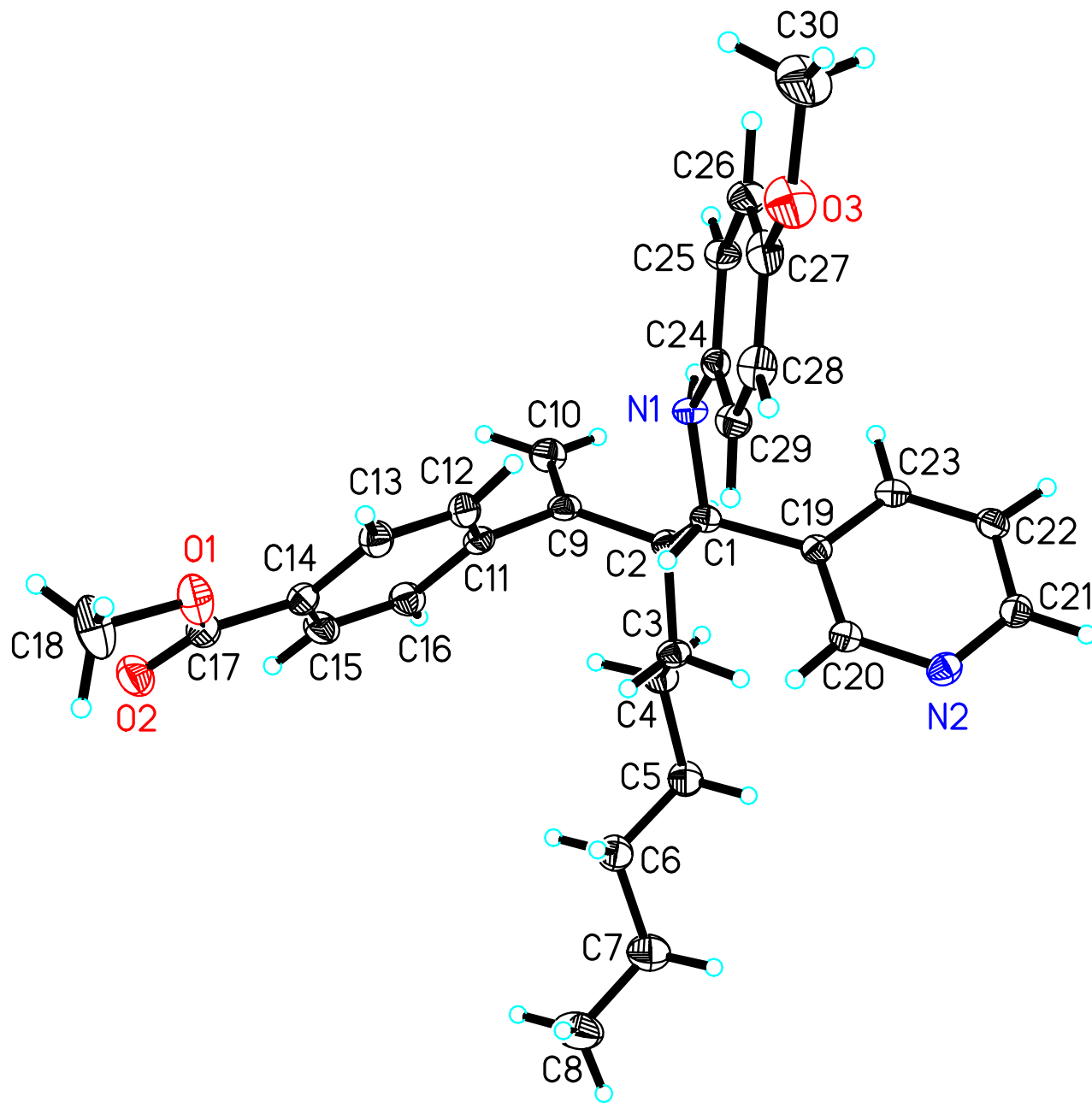

Figure S1. Thermal ellipsoid diagram for compound $( \pm)-(R, R)-\mathbf{1 0}$. Ellipsoids are drawn at the $50 \%$ probability level. 